\title{
Three-dimensional non-linear magnetohydrodynamic modeling of massive gas injection triggered disruptions in JET
}

\section{Cite as: Phys. Plasmas 22, 062509 (2015); https://doi.org/10.1063/1.4922846 \\ Submitted: 24 March 2015. Accepted: 28 May 2015. Published Online: 24 June 2015 \\ A. Fil, E. Nardon, M. Hoelzl, G. T. A Huijsmans, F. Orain, M. Becoulet, P. Beyer, G. Dif-Pradalier, R. Guirlet, H. R. Koslowski, M. Lehnen, J. Morales, S. Pamela, C. Passeron, C. Reux, F. Saint-Laurent, and JET Contributors \\ ARTICLES YOU MAY BE INTERESTED IN \\ Status of research toward the ITER disruption mitigation system \\ Physics of Plasmas 22, 021802 (2015); https://doi.org/10.1063/1.4901251 \\ Theory of tokamak disruptions \\ Physics of Plasmas 19, 058101 (2012); https://doi.org/10.1063/1.3703327 \\ Thermal quench mitigation and current quench control by injection of mixed species shattered pellets in DIII-D \\ Physics of Plasmas 23, 062516 (2016); https://doi.org/10.1063/1.4954389 \\ 里 \\ View Online

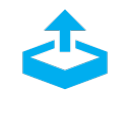 \\ Export Citation

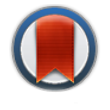

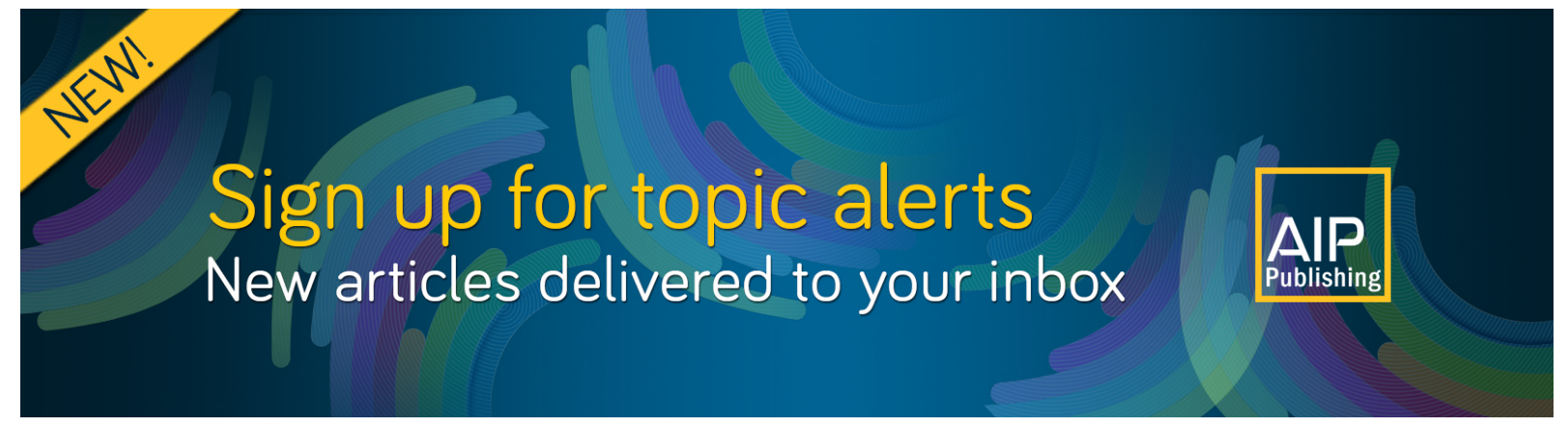




\title{
Three-dimensional non-linear magnetohydrodynamic modeling of massive gas injection triggered disruptions in JET
}

\author{
A. Fil, ${ }^{1}$ E. Nardon, ${ }^{1}$ M. Hoelzl, ${ }^{2}$ G. T. A Huijsmans, ${ }^{3}$ F. Orain, ${ }^{2}$ M. Becoulet, ${ }^{1}$ P. Beyer, ${ }^{4}$ \\ G. Dif-Pradalier, ${ }^{1}$ R. Guirlet, ${ }^{1}$ H. R. Koslowski, ${ }^{5}$ M. Lehnen, ${ }^{3}$ J. Morales, ${ }^{1}$ S. Pamela, ${ }^{6}$ \\ C. Passeron, ${ }^{1}$ C. Reux, ${ }^{1}$ F. Saint-Laurent, ${ }^{1}$ and JET Contributors ${ }^{6, a)}$ \\ ${ }^{1}$ CEA, IRFM, F-13108 Saint-Paul-Lez-Durance, France \\ ${ }^{2}$ Max Planck Institute for Plasma Physics, Boltzmannstr. 2, 85748 Garching b. M., Germany \\ ${ }^{3}$ ITER Organization, Route de Vinon sur Verdon, 13115 Saint Paul Lez Durance, France \\ ${ }^{4}$ Aix-Marseille University, CNRS, PIIM UMR 7345, 13397 Marseille Cedex 20, France \\ ${ }^{5}$ Forschungszentrum Juelich GmbH, Institute of Energy and Climate ResearchPlasma Physics (IEK-4), \\ Trilateral Euregio Cluster, 52425 Juelich, Germany \\ ${ }^{6}$ EUROfusion Consortium, JET, Culham Science Centre, Abingdon OX14 3DB, United Kingdom
}

(Received 24 March 2015; accepted 28 May 2015; published online 24 June 2015)

JOREK 3D non-linear MHD simulations of a $D_{2}$ Massive Gas Injection (MGI) triggered disruption in JET are presented and compared in detail to experimental data. The MGI creates an overdensity that rapidly expands in the direction parallel to the magnetic field. It also causes the growth of magnetic islands $(m / n=2 / 1$ and 3/2 mainly) and seeds the $1 / 1$ internal kink mode. O-points of all island chains (including 1/1) are located in front of the MGI, consistently with experimental observations. A burst of MHD activity and a peak in plasma current take place at the same time as in the experiment. However, the magnitude of these two effects is much smaller than in the experiment. The simulated radiation is also much below the experimental level. As a consequence, the thermal quench is not fully reproduced. Directions for progress are identified. Radiation from impurities is a good candidate. (C) 2015 AIP Publishing LLC. [http://dx.doi.org/10.1063/1.4922846]

\section{INTRODUCTION}

A tokamak disruption ${ }^{1-3}$ is a violent loss of plasma confinement due to the development of a global instability. It comprises two consecutive phases: the Thermal Quench (TQ) when the thermal energy is lost and the Current Quench (CQ) when the plasma current is lost due to the very large resistivity of the cold post-TQ plasma. Typical durations of these phases in present tokamaks are on the order of a millisecond and a few tens of milliseconds, respectively. Disruptions have three types of potentially deleterious effects: heat loads on the Plasma Facing Components (PFC), the formation of Runaway Electron (RE) beams (which can cause extremely intense heat loads when they hit the PFC), and electromagnetic forces on the tokamak structure. All these effects increase with machine size. Disruptions already pose problems in present large tokamaks and have led to the routine use of Disruption Mitigation Systems (DMS) for example on JET and ASDEX Upgrade. In ITER, a DMS will be mandatory. Its design is currently underway and recent overviews on this topic are given in Refs. 4 and 5.

Massive Gas Injection (MGI) is one of the main concepts under consideration for the ITER DMS. MGI aims at spreading heat loads by radiating most of the plasma stored energy, preventing the generation of REs by increasing the electron density and controlling the CQ duration (in order to limit mechanical loads) by controlling the impurity content

\footnotetext{
${ }^{\text {a) }}$ For a complete listing of the JET contributors, refer to F. Romanelli et al., Proceedings of the 25th IAEA Fusion Energy Conference 2014, Saint Petersburg, Russia.
}

and thus the temperature and resistivity of the CQ plasma. A large body of experimental work on MGI exists, including experiments on JET, ${ }^{6-9}$ ASDEX Upgrade, ${ }^{10}$ Tore Supra, ${ }^{11}$ DIII-D,${ }^{12}$ TEXTOR, ${ }^{13}$ Alcator C-MOD,${ }^{14}$ and other devices. Results have shown the capability of MGI to fulfill part of the objectives of the ITER DMS, but not yet all of them. For example, the radiation efficiency of MGI should be higher than $90 \%$ for the ITER baseline scenario, ${ }^{4}$ but this objective has not been reached in JET experiments at high thermal energy fraction $W_{t h} / W_{t o t}{ }^{7}$ In addition, radiation asymmetries should be small in order not to melt the Beryllium first wall. Another topic on which progress remains to be done is RE suppression. For example, recent results on $\mathrm{JET}^{15}$ suggest that MGI may prevent REs only if the gas reaches the plasma before the formation of a runaway beam. This seems inconsistent with a strategy based on two successive material (gas or pellets) injections, the first one to mitigate the thermal loads and the second one to suppress REs, as is envisaged for ITER. ${ }^{4}$ In addition to further experiments, modeling can help overcome these obstacles and provide important input for the design and future operation of the ITER DMS. Due to the variety of timescales and phenomena involved, several types of codes have been applied. For example, axisymmetric $1.5 \mathrm{D}$ integrated modeling codes like DINA $^{16}$ or ASTRA $^{17}$ allow simulating a full disruption in a moderate computational time. However, 3D aspects and in particular 3D MHD activity are a central ingredient of disruption physics which influences many of the effects of disruptions, e.g., radiation asymmetries, heat load deposition patterns, and the creation or losses of REs. ${ }^{18}$ 3D non-linear MHD 
TABLE I. Normalization of quantities in JOREK. Variable names with subscript "SI" denote quantities in SI units, while variables without this subscript are the ones used in JOREK. $n_{0}$ and $\rho_{0}$ are the initial central plasma particle and mass density. The vacuum magnetic permeability is denoted $\mu_{0}$ and the Boltzmann constant $k_{B}$.

\begin{tabular}{|c|c|c|}
\hline$R_{\mathrm{SI}}(\mathrm{m})$ & $=R$ & Major radius \\
\hline$Z_{\mathrm{SI}}(\mathrm{m})$ & $=Z$ & Vertical coordinate \\
\hline $\boldsymbol{B}_{\mathrm{SI}}(\mathrm{T})$ & $=\boldsymbol{B}$ & Magnetic field vector; see Eq. (9) \\
\hline$\psi_{\mathrm{SI}}\left(\mathrm{T} \cdot \mathrm{m}^{2}\right)$ & $=\psi$ & Poloidal magnetic flux \\
\hline$j_{\phi, \mathrm{SI}}\left(\mathrm{A} \cdot \mathrm{m}^{-2}\right)$ & $=-j /\left(R \mu_{0}\right)$ & Toroidal current density; $j_{\phi, \mathrm{SI}}=\boldsymbol{j}_{\mathrm{SI}} \cdot \hat{\boldsymbol{e}}_{\phi}$ \\
\hline$n_{\mathrm{SI}}\left(\mathrm{m}^{-3}\right)$ & $=\rho n_{0}$ & Particle density \\
\hline$\rho_{\mathrm{SI}}\left(\mathrm{kg} \cdot \mathrm{m}^{-3}\right)$ & $=\rho \rho_{0}$ & Mass density $=$ ion mass $\times$ particle density \\
\hline$T_{\mathrm{SI}}(\mathrm{K})$ & $=T /\left(k_{B} \mu_{0} n_{0}\right)$ & Temperature $=$ electron + ion temperature \\
\hline$p_{\mathrm{SI}}\left(N \cdot \mathrm{m}^{-2}\right)$ & $=\rho T / \mu_{0}$ & Plasma pressure \\
\hline$v_{\mathrm{SI}}\left(\mathrm{m} \cdot \mathrm{s}^{-1}\right)$ & $=\boldsymbol{v} / \sqrt{\mu_{0} \rho_{0}}$ & Velocity vector; see Eq. (10) \\
\hline$v_{\|, \mathrm{SI}}\left(\mathrm{m} \cdot \mathrm{s}^{-1}\right)$ & $=v_{\|} \cdot B_{\mathrm{SI}} / \sqrt{\mu_{0} \rho_{0}}$ & Parallel velocity component, where $B_{\mathrm{SI}}=\left|\boldsymbol{B}_{\mathrm{SI}}\right|$ \\
\hline$u_{\mathrm{SI}}\left(\mathrm{m} \cdot \mathrm{s}^{-1}\right)$ & $=u / \sqrt{\mu_{0} \rho_{0}}$ & Velocity stream function \\
\hline$\omega_{\phi, \mathrm{SI}}\left(\mathrm{m}^{-1} \cdot \mathrm{s}^{-1}\right)$ & $=\omega / \sqrt{\mu_{0} \rho_{0}}$ & Toroidal vorticity; see Eq. (4) \\
\hline$t_{\mathrm{SI}}(\mathrm{s})$ & $=t \cdot \sqrt{\mu_{0} \rho_{0}}$ & Time \\
\hline$\gamma_{\mathrm{SI}}\left(\mathrm{s}^{-1}\right)$ & $=\gamma / \sqrt{\mu_{0} \rho_{0}}$ & Growth rate; $\gamma_{\mathrm{SI}}=\ln \left(E_{\mathrm{SI}}\left(t_{2}\right) / E_{\mathrm{SI}}\left(t_{1}\right)\right) /\left(2 \Delta t_{\mathrm{SI}}\right) ;$ Energy $E_{\mathrm{SI}}(J)$ \\
\hline$\eta_{\mathrm{SI}}(\Omega \cdot \mathrm{m})$ & $=\eta \cdot \sqrt{\mu_{0} / \rho_{0}}$ & Resistivity \\
\hline$\mu_{\mathrm{SI}}\left(\mathrm{kg} \cdot \mathrm{m}^{-1} \cdot \mathrm{s}^{-1}\right)$ & $=\mu \cdot \sqrt{\mu_{0} / \rho_{0}}$ & Dynamic viscosity \\
\hline$D_{\mathrm{SI}}\left(\mathrm{m}^{2} \cdot \mathrm{s}^{-1}\right)$ & $=D / \sqrt{\mu_{0} \rho_{0}}$ & Particle diffusivity $(\|$ or $\perp)$ \\
\hline$\kappa_{\mathrm{SI}}\left(\mathrm{m}^{-1} \cdot \mathrm{s}^{-1}\right)$ & $=\kappa \cdot n_{0} / \sqrt{\mu_{0} \rho_{0}}$ & Heat diffusivity $(\|$ or $\perp)$, where $\chi_{\mathrm{SI}}\left(\mathrm{m}^{2} \cdot \mathrm{s}^{-1}\right)=\kappa_{\mathrm{SI}} / n_{\mathrm{SI}}$ \\
\hline$S_{i o n, S I}\left(\mathrm{~m}^{-3} \cdot \mathrm{s}^{-1}\right)$ & $=S_{\text {rec }} /\left(\sqrt{\mu_{0} \rho_{0}} n_{0}\right)$ & Ionisation rate coefficient \\
\hline$\alpha_{\text {rec }, S I}\left(\mathrm{~m}^{-3} \cdot \mathrm{s}^{-1}\right)$ & $=\alpha_{\text {rec }} /\left(\sqrt{\mu_{0} \rho_{0}} n_{0}\right)$ & Recombination rate coefficient \\
\hline$E_{\text {ion }, S I}(J)$ & $=\xi_{\text {ion }} /\left(\frac{2}{3} \mu_{0} n_{0}\right)$ & Ionisation energy \\
\hline$L_{\text {lines } / \text { brem }, S I}\left(\mathrm{~W} \cdot \mathrm{m}^{3}\right)$ & $=L_{\text {lines } / \text { brem }} /\left(\frac{2}{3} \mu_{0} \sqrt{\mu_{0} \rho_{0}} n_{0}^{2}\right)$ & Radiation rate coefficients (lines or bremsstrahlung) \\
\hline
\end{tabular}

codes have been applied to disruption simulations for several decades (see Refs. 19 and 1-3, and references therein) with gradually increasing accuracy thanks to increasing computational resources. Recent work includes M3D simulations which were mainly focused on the vertical displacement of the plasma and the halo current ${ }^{20}$ and NIMROD simulations of MGI-triggered disruptions. ${ }^{21-25}$

The present paper describes simulations of an MGItriggered disruption in JET with another 3D non-linear MHD code, JOREK. ${ }^{26}$ So far, JOREK has been applied mainly to edge localised mode modeling. ${ }^{27-30}$ Work on MGI-triggered disruptions has been initiated by C. Reux et al. for Tore Supra with a simplified MGI model. ${ }^{31,32}$ Recently, this model has been applied to JET and has allowed simulating the TQ. ${ }^{33}$ After this first step, the objective is to quantitatively validate the model. For this purpose, the MGI model has been improved in order to treat correctly the atomic physics for deuterium $\left(D_{2}\right)$. $D_{2}$ MGI is not considered as a good option for disruption mitigation due to its low radiation efficiency. ${ }^{14}$ Instead, noble gases like Ar or $\mathrm{Ne}$, possibly mixed with $D_{2}$, are used in JET and ASDEX Upgrade for disruption mitigation and considered for the ITER DMS. However, simulating a $D_{2}$ MGI-triggered disruption constitutes a good intermediate step due to the relatively simple atomic physics, before moving to simulations of heavier gases.

This paper is constructed as follows: Section II describes the JOREK model adapted for MGI simulations; Section III presents the experimental pulse chosen for the simulations; Section IV details the simulation set-up; Section V presents the simulation results and their comparison to the experiment; and finally, Section VI summarizes and gives perspectives for future work.

\section{THE JOREK CODE ADAPTED FOR MGI SIMULATIONS}

JOREK is a non-linear MHD code in 3D toroidal geometry including the X-point and the Scrape-Off Layer (SOL) in the computational domain. We use here the so-called "model 500" of JOREK, which is single-fluid large aspect ratio reduced MHD with an equation for neutral density and additional terms related to atomic physics in several equations.

Eight physical variables (normalized as summarized in Table I) are evolved in time: poloidal flux $\psi$, toroidal current density $j$, poloidal flow potential $u$, toroidal vorticity $\omega$, plasma mass density $\rho$, total (ion + electron) pressure $\rho T$, parallel velocity $v_{\|}$(in this paper, the adjectives "parallel" and "perpendicular" refer to the direction of the unperturbed magnetic field), and neutral mass density $\rho_{n}$, according to the following differential equations:

$$
\begin{gathered}
\frac{\partial \psi}{\partial t}=\eta(T) \Delta^{*} \psi-R[u, \psi]-F_{0} \frac{\partial u}{\partial \phi}, \\
j=\Delta^{*} \psi,
\end{gathered}
$$

$$
\begin{aligned}
R \boldsymbol{\nabla} & \cdot\left(R^{2} \rho \nabla_{p o l} \frac{\partial u}{\partial t}\right) \\
= & \frac{1}{2}\left[R^{2}\left|\nabla_{p o l} u\right|^{2}, R^{2} \rho\right]+\left[R^{4} \rho \omega, u\right]+[\psi, j]-\frac{F_{0}}{R} \frac{\partial j}{\partial \phi} \\
& +\left[\rho T, R^{2}\right]+R \mu(T) \nabla^{2} \omega \\
& +\boldsymbol{\nabla} \cdot\left(\left(\rho \rho_{n} S_{i o n}(T)-\rho^{2} \alpha_{r e c}(T)\right) R^{2} \nabla_{p o l} u\right)
\end{aligned}
$$




$$
\begin{aligned}
& \omega=\nabla_{p o l}^{2} u=\frac{1}{R} \frac{d}{d R}\left(R \frac{d u}{d R}\right)+\frac{d^{2} u}{d Z^{2}}, \\
& \frac{\partial \rho}{\partial t}=-\boldsymbol{\nabla} \cdot(\rho \boldsymbol{v})+\boldsymbol{\nabla} \cdot\left(D_{\perp} \boldsymbol{\nabla}_{\perp} \rho+D_{\|} \boldsymbol{\nabla}_{\|} \rho\right) \\
& +\rho \rho_{n} S_{i o n}(T)-\rho^{2} \alpha_{\text {rec }}(T), \\
& \frac{\partial(\rho T)}{\partial t}=-\boldsymbol{v} \cdot \boldsymbol{\nabla}(\rho T)-\gamma \rho T \nabla \cdot \boldsymbol{v}+\boldsymbol{\nabla} \cdot\left(\kappa_{\perp} \boldsymbol{\nabla}_{\perp} T+\kappa_{\|} \boldsymbol{\nabla}_{\|} T\right) \\
& +\frac{2}{3 R^{2}} \eta_{\text {Spitzer }}(T) j^{2}-\xi_{\text {ion }} \rho \rho_{n} S_{\text {ion }}(T) \\
& -\rho \rho_{n} L_{\text {lines }}(T)-\rho^{2} L_{\text {brem }}(T) \text {, } \\
& \rho B^{2} \frac{\partial v_{\|}}{\partial t}=-\rho \frac{F_{0}}{2 R^{2}} \frac{\partial\left(B^{2} v_{\|}^{2}\right)}{\partial \phi}-\frac{\rho}{2 R}\left[B^{2} v_{\|}^{2}, \psi\right]-\frac{F_{0}}{R^{2}} \frac{\partial(\rho T)}{\partial \phi} \\
& +\frac{1}{R}[\psi, \rho T]+B^{2} \mu_{\|}(T) \nabla_{p o l}^{2} v_{\|} \\
& +\left(\rho^{2} \alpha_{r e c}(T)-\rho \rho_{n} S_{i o n}(T)\right) B^{2} v_{\|}, \\
& \frac{\partial \rho_{n}}{\partial t}=\boldsymbol{\nabla} \cdot\left(\boldsymbol{D}_{n}: \nabla \rho_{n}\right)-\rho \rho_{n} S_{i o n}(T)+\rho^{2} \alpha_{r e c}(T)+S_{n},
\end{aligned}
$$

where $(R, Z, \phi)$ is a direct toroidal coordinate system, $\nabla_{p o l}$ denotes the del-operator in the poloidal plane, the Poisson brackets are defined as $[f, g]=\frac{\partial f}{\partial R} \frac{\partial g}{\partial Z}-\frac{\partial f}{\partial Z} \frac{\partial g}{\partial R}$, and the parallel gradient as $\nabla_{\|}=\boldsymbol{b}(\boldsymbol{b} \cdot \nabla)$, where $\boldsymbol{b}=\boldsymbol{B} /|\boldsymbol{B}|$.

The magnetic field is defined as

$$
\boldsymbol{B}=F_{0} \nabla \phi+\nabla \psi \times \nabla \phi,
$$

with the diamagnetic function $F_{0}$ considered constant.

The velocity vector is defined as

$$
\boldsymbol{v}=\boldsymbol{v}_{E \times B}+v_{\|} \boldsymbol{B}=R^{2} \nabla \phi \times \nabla u+v_{\|} \boldsymbol{B} .
$$

$S_{i o n}$ and $\alpha_{\text {rec }}$ designate, respectively, the ionization and recombination rate coefficients for deuterium, parameterized according to Refs. 34 and 35 as

$$
\begin{gathered}
S_{\text {ion }}\left(T_{e}\right)=\left\langle\sigma_{\text {ion }} v\right\rangle=0.2917 \times 10^{-13}\left(\frac{13.6}{T_{e}}\right)^{0.39} \\
\times \frac{1}{0.232+\frac{13.6}{T_{e}}} \exp \left(-\frac{13.6}{T_{e}}\right), \\
\alpha_{\text {rec }}\left(T_{e}\right)=\left\langle\sigma_{\text {rec }} v\right\rangle=0.7 \times 10^{-19}\left(\frac{13.6}{T_{e}}\right)^{\frac{1}{2}},
\end{gathered}
$$

where $S_{i o n}$ and $\alpha_{r e c}$ are in $\mathrm{m}^{3} / \mathrm{s}$ and $T_{e}$ is in $\mathrm{eV}$. In the code, we assume that $T_{e}=T / 2$, so that $T_{i}=T_{e}$.

$\xi_{\text {ion }}$ is the normalized ionization energy of a $D$ atom, which is considered to be $13.6 \mathrm{eV}$. In the present work, we neglect the bond-dissociation energy of $D_{2}$ molecules. Assuming that this energy is the same as for $H_{2}$ molecules, i.e., about $5 \mathrm{eV}$, this seems reasonable in the sense that it is smaller than the ionisation energy of two $D$ atoms by a factor of about 5 .

$L_{\text {lines }}$ and $L_{\text {brem }}$ designate the line and bremsstrahlung radiation rate coefficients. A fit of ADAS data ${ }^{36}$ is used for line radiation and bremsstrahlung is parameterized according to Ref. 37. It is interesting to compare the energy sink rates related to atomic physics which appear in the energy equation (Eq. (6)), i.e., $\xi_{\text {ion }} S_{\text {ion }}, L_{\text {lines }}$ and $L_{\text {brem }}$. This is done in Figure 1 . For $T_{e}>10 \mathrm{eV}$, ionization slightly dominates line radiation, while below $10 \mathrm{eV}$, line radiation is dominant. The bremsstrahlung rate coefficient is roughly 5 orders of magnitude smaller than the other two rates, but, of course, it should not be compared directly since in Eq. (6) it is multiplied by $\rho^{2}$ whereas the other rates are multiplied by $\rho \rho_{n}$. The relative influence of these terms will be discussed in details in section $\mathrm{VC}$.

The resistivity $\eta$ which appears in the induction equation (Eq. (1)) is typically increased in JOREK simulations compared to the Spitzer value in order to thicken the current sheets which otherwise would be too thin to be resolved (for the same purpose, a hyper-resistivity term, not shown in Eq. (1), may be used). However, in the energy equation (Eq. (6)), the Joule heating term $\eta_{\text {Spitzer }} j^{2}$ uses the Spitzer resistivity in order not to alter the energy balance.

$S_{n}$ is a volumetric neutral source term used to simulate the influx of gas from the MGI. Its parametrization will be described in detail in Sec. IV.

One limit of the model is the purely diffusive treatment of neutral transport, which does not stem directly from first principles. This point must be kept in mind in the interpretation of the results presented in Sec. V.

\section{DESCRIPTION OF THE EXPERIMENT}

We simulate JET pulse 86887 . This is an Ohmic $D$ plasma pulse with $B_{t}=2 \mathrm{~T}, I_{p}=2 \mathrm{MA}, q_{95}=2.9$ in which a disruption was MGI-triggered on a "healthy" plasma by activating the Disruption Mitigation Valve number 2 (DMV2), pre-loaded with $D_{2}$ at $5 \mathrm{bar}\left(5 \times 10^{5} \mathrm{~Pa}\right)$, at $t=61.013 \mathrm{~s}$. The times given below are relative to this time. The volume of the DMV2 reservoir is $10^{-3} \mathrm{~m}^{3}$, and its temperature is about $293 \mathrm{~K}$, so it initially contains about $1.2 \times 10^{23} D_{2}$ molecules, which represents roughly 10 times more $D$ nuclei than initially present in the plasma. Electron density $n_{e}$ and

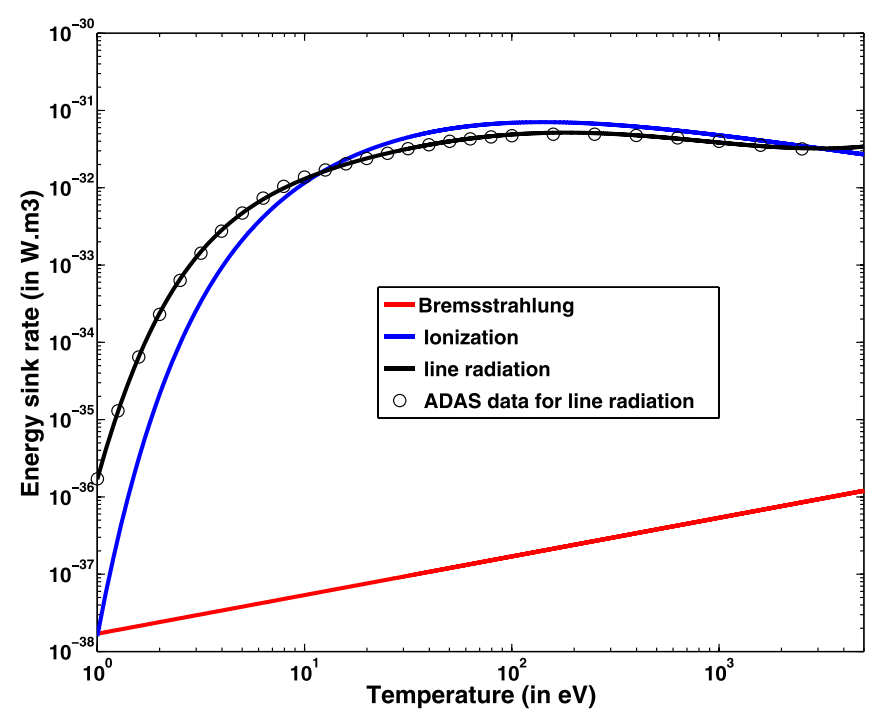

FIG. 1. Energy sink rates related to atomic physics which appear in Eq. (6): $\xi_{\text {ion }} S_{\text {ion }}, L_{\text {lines }}$, and $L_{\text {brem }}$. 

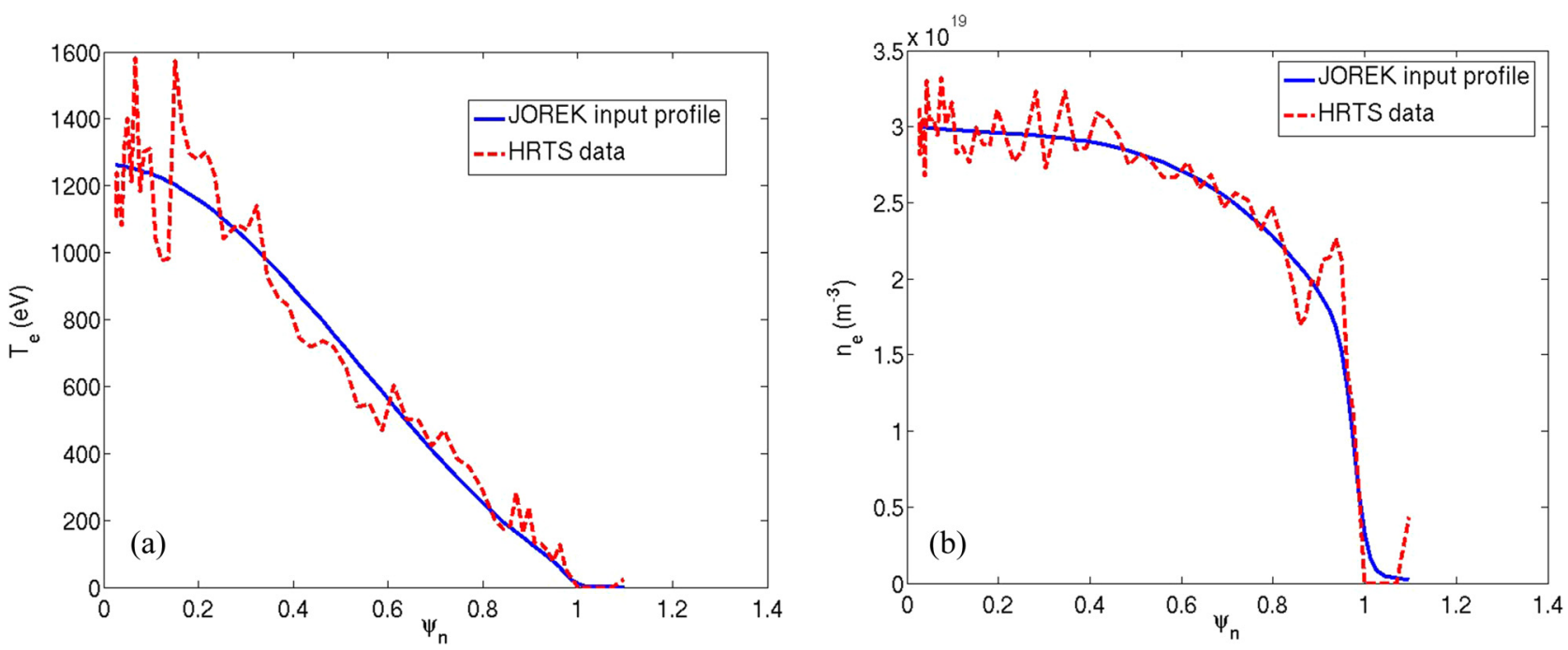

FIG. 2. Experimental $T_{e}$ and $n_{e}$ profiles from high resolution Thomson scattering (dashed lines) and fits of these profiles used as initial conditions in the JOREK simulations (plain lines).

temperature $T_{e}$ profiles measured by High Resolution Thomson Scattering (HRTS) just before the MGI, together with fits of these profiles used as initial conditions in the JOREK simulations, are shown in Figure 2. Central values are $n_{e}=3 \times 10^{19} \mathrm{~m}^{-3}$ and $T_{e}=1.2 \mathrm{keV}$.

Figure 3 shows an overview of the disruption phase. First effects of the MGI are visible from about $2 \mathrm{~ms}$ in the form of increases in the line integrated density, radiated power $P_{\text {rad }}$, and magnetic fluctuations, and decreases in $I_{p}$ and the central Soft-X Ray (SXR) signal. These effects intensify in time, especially the drop in SXR, until at about $12 \mathrm{~ms}$; the SXR signal quickly drops to zero and a burst of MHD activity, a peak on $I_{p}$ and, a few milliseconds later, a peak on $P_{\text {rad }}$ are observed. It is interesting to note that most of the drop of the central SXR signal occurs before the burst of

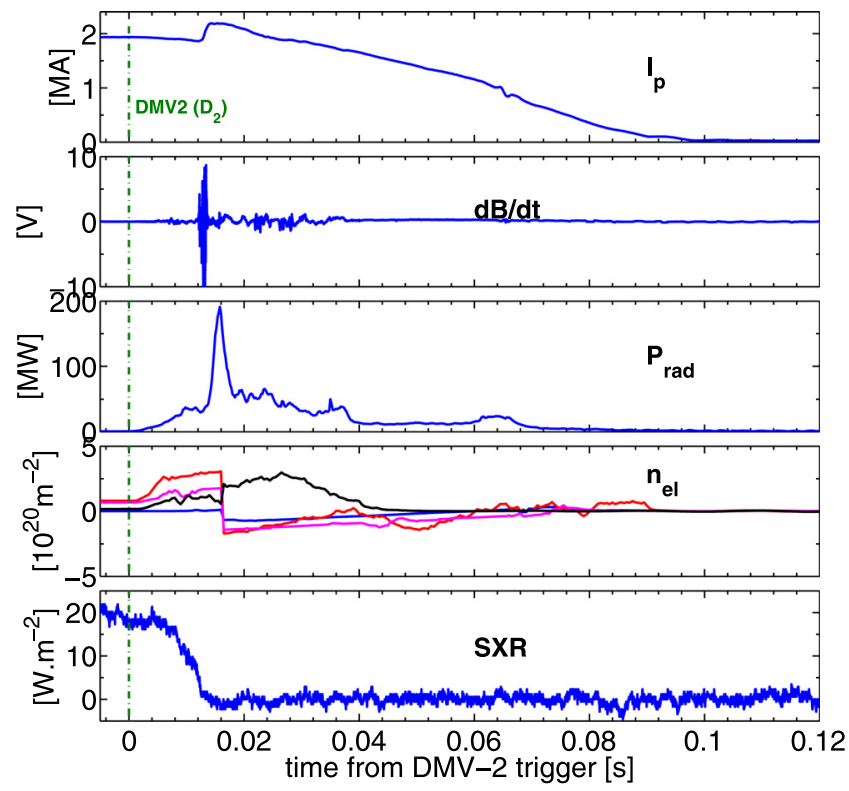

FIG. 3. Experimental time traces, from top to bottom: plasma current $I_{p}$, magnetic fluctuations from Mirnov coil H302, radiated power from bolometry, line integrated density from interferometry (valid until about $10 \mathrm{~ms}$ ), and soft X-rays signal from a central chord. The time origin corresponds to the DMV2 trigger.
MHD activity and on a rather slow timescale (on the order of $10 \mathrm{~ms}$ ). The CQ ensues and lasts about $80 \mathrm{~ms}$. We note that magnetic fluctuations and $P_{\text {rad }}$ remain at a substantial level during the first $20 \mathrm{~ms}$ of this CQ.

\section{SIMULATIONS SET-UP}

\section{A. Initialization}

The JOREK simulations start by solving the GradShafranov equation, using input from the equilibrium reconstructed by EFIT (Equilibrium FITting) at $t=60.9 \mathrm{~s}$ (i.e., about $100 \mathrm{~ms}$ before the MGI). More precisely, the EFIT poloidal flux $\psi$ is used as a boundary condition in JOREK. Concerning $p^{\prime}$ and $f f^{\prime}$, the EFIT profiles are not directly used since they do not take kinetic measurements into account. Instead, HRTS $n_{e}$ and $T_{e}$ measurements (see Figure 2) are used to compute the pressure profile, assuming $T_{i}=T_{e}$. Mapping this profile on the EFIT $\psi$ and deriving with respect to $\psi$ provides $p^{\prime}$. The $f f^{\prime}$ profile is then adjusted so that the flux surface averaged toroidal current density profile $j_{\text {mean }}\left(\psi_{n}\right)=\left\langle j_{\phi} / R\right\rangle /\langle 1 / R\rangle$ (where $\psi_{n}$ is the normalized EFIT $\psi$, equal to 0 on the magnetic axis and 1 at the last closed flux surface) remains close to the one provided by EFIT. For this purpose, the relationship between $p^{\prime}, f f^{\prime}$, and $j_{\text {mean }}$ provided by the flux surface averaged Grad-Shafranov equation is used.

After solving the Grad-Shafranov equation, JOREK constructs a flux surface aligned grid in the poloidal plane which is shown in Figure 4. Typically, about 100 points in the radial direction and 200 in the poloidal direction are used. 2D Bezier finite elements are used for the poloidal plane. ${ }^{38}$ Concerning the toroidal discretization, JOREK uses Fourier harmonics. The simulations presented here include all harmonics from $n=0$ to $n=5$ (from $n=0$ to $n=10$ for the simulation with $q_{0}=0.94$ ).

Starting from the Grad-Shafranov solution, time evolution begins. Before turning on the MGI, the plasma is let to evolve for about $1000 \times \tau_{A}$ in order for SOL flows to establish, i.e., for the Bohm boundary conditions imposed at 


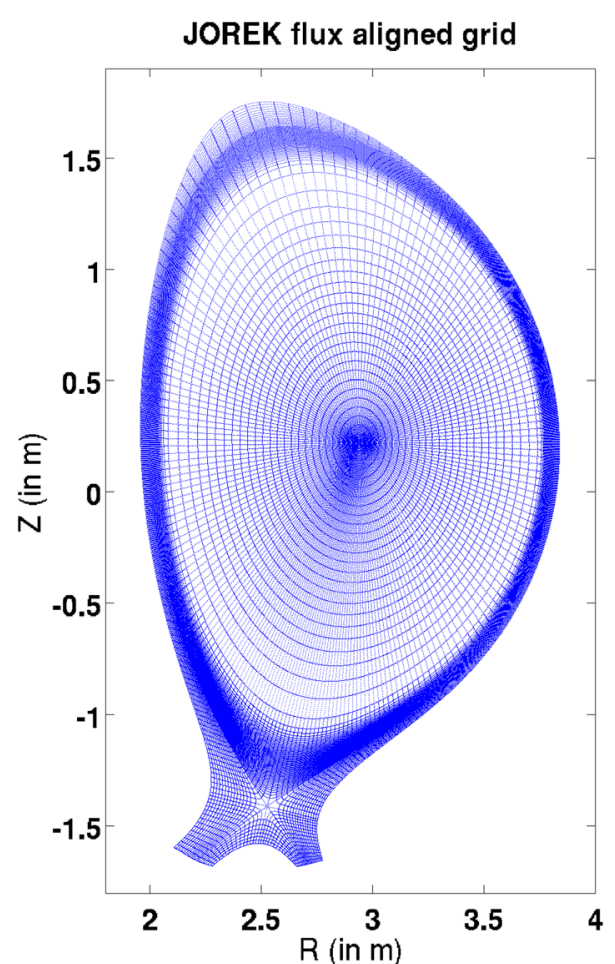

FIG. 4. JOREK flux surface aligned grid built from 200 poloidal chords and 81 closed, 15 open and 8 private flux surfaces. In total, the grid contains about 20000 cells in the poloidal plane.

the target plates to propagate and for flow equilibrium to be reached.

It should be mentioned that the initial toroidal rotation profile is set to 0 in these simulations. The rotation is probably small in this Ohmic scenario, but no measurements are available.

\section{B. Massive gas injection}

Then, the MGI is triggered by turning on the volumetric source term $S_{n}$ appearing in Eq. (8). The following expression is used:

$$
S_{n}=\frac{d M_{n}}{d t}(t) \cdot \frac{f(R, Z, \phi)}{\int f d V},
$$

with the spatial shape of the source set as

$$
\begin{aligned}
f= & \exp \left(-\frac{\left(R-R_{M G I}\right)^{2}+\left(Z-Z_{M G I}\right)^{2}}{\Delta r_{M G I}^{2}}\right) \\
& \times \exp \left(-\left(\frac{\phi-\phi_{M G I}}{\Delta \phi_{M G I}}\right)^{2}\right) .
\end{aligned}
$$

Here, $\left(R_{M G I}, Z_{M G I}, \phi_{M G I}\right)=(3.8 \mathrm{~m}, 0.28 \mathrm{~m}, 4.51 \mathrm{rad})$ is the position where neutrals from DMV2 are assumed to be delivered into the plasma and $\Delta r_{M G I}=4 \mathrm{~cm}$ and $\Delta \phi_{M G I}=0.6 \mathrm{rad}$ are the assumed poloidal and toroidal extensions of the neutral source. Note that the value of $\Delta \phi_{M G I}$ is constrained by the number of toroidal harmonics $n_{\text {tor }}$ included in the simulation, the real value being probably smaller than $0.6 \mathrm{rad}$.
The normalization by $\int f d V$ in expression (13) ensures that the total mass of neutrals injected per time unit is equal to $\frac{d M_{n}}{d t}$. The parameterization of $\frac{d M_{n}}{d t}$ is based on laboratory experiments and modeling of the DMV reported in Ref. 13. After the valve opening, the gas travels inside a guiding tube of length $L_{\text {tube }}=2.36 \mathrm{~m}$ and cross-sectional area $A_{\text {tube }}=1.8$ $\times 10^{-2} \mathrm{~m}^{2}$, which is much larger than the valve orifice area. It is shown in Ref. 13 that this situation is well described with the 1D Euler equations, whose solution is a so-called "rarefaction wave." The forefront of this wave travels at a velocity of $3 \cdot c_{s}$, where $c_{s}$ is the gas sound speed at the reservoir temperature. In the present case, $c_{s}=923 \mathrm{~m} / \mathrm{s}$, and it therefore takes $t_{0}=\frac{L_{\text {tube }}}{3 c_{s}} \simeq 0.9 \mathrm{~ms}$ for the first gas particles to arrive at the exit of the tube and enter the vacuum vessel through the midplane port of Octant 3.

$\frac{d N_{n}}{d t}=\frac{1}{m_{D_{2}}} \frac{d M_{n}}{d t}$ is represented in Figure 5. Before $t=t_{0}$, $\frac{d M_{n}}{d t}=0$. Then, for $t_{0}<t<t_{1}$, the mass of gas entering the vessel per unit time according to Ref. 13 is

$$
\begin{aligned}
\frac{d M_{n}}{d t}(t)= & \rho_{0_{D M V 2}} A_{\text {tube }} K L_{\text {tube }} \frac{m^{m}}{(m+1)^{m+1}} \\
& \times \sum_{k=0}^{m+1} \frac{(-1)^{k-1}(m+1) !}{(m-k+1) ! k !}(k-1)\left(\frac{L_{\text {tube }}}{c_{s} m}\right)^{k-1}(t)^{-k},
\end{aligned}
$$

where $\rho_{0_{D M V 2}}=m_{D_{2}} P_{D M V 2} V_{D M V 2} /\left(k_{B} T_{D M V 2}\right)$ is the initial mass density in the DMV2 reservoir, $K$ is a factor calculated from laboratory experiments which depends mainly on the ratio of the valve orifice area to the tube area $A_{\text {tube }}$, and $m=2 /(\gamma-1)$, where $\gamma=c_{p} / c_{v}$ is the ratio of specific heats ( $m=5$ for $D_{2}$ ). $t_{1}$ corresponds to the moment when the time integral of $\frac{d M_{n}}{d t}$ is equal to the mass of gas initially contained in the reservoir and logically, $\frac{d M_{n}}{d t}=0$ for $t>t_{1}$ (this sharp cut at $t=t_{1}$ is an approximation of the model, in reality $\frac{d M_{n}}{d t}$ is continuous).

\section{Other input parameters}

In the simulations presented here, the initial value of the resistivity $\eta$ at the centre of the plasma is $\eta_{0}=10^{-8}-10^{-7}$

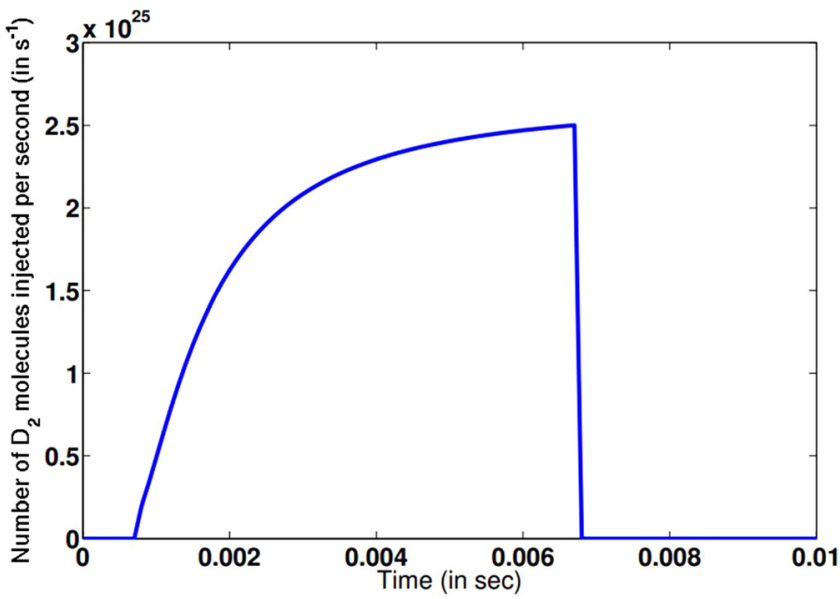

FIG. 5. Number of $D_{2}$ molecules injected per time unit into the JET vacuum vessel by DMV2 pre-loaded with $D_{2}$ at 5 bar, according to Ref. 13 . 
in JOREK units, i.e., $\eta_{0, S I}=3.5 \times 10^{-8}-3.5 \times 10^{-7} \Omega$.m. The experimental Spitzer value ${ }^{37}$ is about $2 \times 10^{-8} \Omega$.m, i.e., a factor $175-175$ smaller than the simulation value. The temperature dependency of $\eta$ is taken into account in JOREK by using $\eta=\eta_{0} \cdot\left(T_{0} / T\right)^{3 / 2}$, where $T_{0}$ is the initial temperature at the centre of the plasma. A rather large hyperresistivity is also used in these simulations for numerical stability purposes, whose influence will be studied in future work.

The parallel heat conductivity used in the simulations is $\kappa_{\|_{0}}=800$ in JOREK units, i.e., $\kappa_{\| 0, S I}=6.7 \times 10^{28} \mathrm{~m}^{-1} \mathrm{~s}^{-1}$. The experimental Spitzer-Härm value ${ }^{37}$ is $6.9 \times 10^{29} \mathrm{~m}^{-1} \mathrm{~s}^{-1}$, i.e., a factor of 10 larger than in the simulation. Similar to the resistivity, $\kappa_{\|}$depends on the temperature: $\kappa_{\|}=\kappa_{\|_{0}}$. $\left(T / T_{0}\right)^{5 / 2}$. The perpendicular heat conductivity is $\kappa_{\perp_{0}}=$ $5 \times 10^{-7}$ in JOREK units, i.e., $\kappa_{\perp 0, S I}=4.2 \times 10^{19} \mathrm{~m}^{-1} \mathrm{~s}^{-1}$, which corresponds to a $\chi_{\perp}$ typical of turbulent transport (of the order of $1 \mathrm{~m}^{2} \cdot \mathrm{s}^{-1}$ ).

For the viscosity, we use, in JOREK units, $\mu=10^{-6}$ and $\mu_{\|}=10^{-4}$, i.e., $\mu_{S I}=2.8 \times 10^{-7} \mathrm{~kg} \cdot \mathrm{m}^{-1} \cdot \mathrm{s}^{-1}$ and $\mu_{\|, S I}=2.8 \times 10^{-5} \mathrm{~kg} \cdot \mathrm{m}^{-1} \cdot \mathrm{s}^{-1}$, and a temperature dependency of the perpendicular viscosity is taken into account, using $\mu_{\perp}=\mu_{0_{\perp}} \cdot\left(T / T_{0}\right)^{-3 / 2}$. Typical particle diffusivities used in the simulations are $D_{n}=10^{-2}, D_{\perp}=10^{-5}$, and $D_{\|}=10^{-2}$ in JOREK units, i.e., $D_{n, S I}=2.8 \times 10^{4} \mathrm{~m}^{2} / \mathrm{s}$, $D_{\perp, S I}=28 \mathrm{~m}^{2} / \mathrm{s}$ and $D_{\|, S I}=2.8 \times 10^{4} \mathrm{~m}^{2} / \mathrm{s}$. The choice of these values is dictated mainly by numerical stability reasons. Indeed, particle diffusion tends to smooth gradients and helps prevent numerical instabilities. In the absence of a first principles model for neutrals transport, it is not clear what a realistic value of $D_{n}$ would be (in fact, a diffusive model may not even be appropriate). As for $D_{\perp}$, a typical value representative of turbulent transport would be $1 \mathrm{~m}^{2} / \mathrm{s}$, a factor of 28 smaller than in the simulation. Finally, $D_{\|}$has no physical origin and is used only for numerical stability reasons. Efforts are currently made by the JOREK community in order to overcome these numerical issues, including generalized finite elements, Taylor-Galerkin stabilization, and an improved treatment of the grid center. Recently, simulations with $D_{\|}=0$ have been run without displaying numerical instabilities.

\section{Computational resources}

These simulations have been run on the HELIOS (from IFERC-CSC) and CCRT-CURIE (from CEA) supercomputers. A typical run on HELIOS uses 36 nodes, and one time step takes around $200 \mathrm{~s}$. Depending on the conditions (e.g., level of MHD activity), the time step typically varies between 1 and 100 in JOREK units (i.e., $0.35-35 \mu$ s). A full simulation represents around $10^{4}$ node-hours.

\section{SIMULATION RESULTS}

Simulation results will now be presented, focusing in Sec. VA on the increase in plasma density caused by the MGI, then in Sec. VB on MHD aspects, and finally in Sec. $\mathrm{VC}$ on radiation aspects.

\section{A. Increase in electron density}

As the MGI is turned on, the neutral density $\rho_{n}$ increases and takes a spatial distribution similar to that of the source $S_{n}$, as shown in Figure 6. After a fast transient increase, $\rho_{n}$ becomes approximately stationary, which indicates that an equilibrium is established between sources, sinks, and transport terms in Eq. (8). The stationary neutral density at the injection location is on the order of $10^{19} \mathrm{~m}^{-3}$.

The ionization of neutrals causes a local increase in plasma density, as can be seen in Figure 7. The density at the location where neutrals are deposited reaches several times $10^{20} \mathrm{~m}^{-3}$. This is accompanied by a cooling of the edge of the plasma, also visible on Figure 7.

Figure 8 shows that the overdensity expands in the parallel direction. In the simulations, parallel diffusion and convection contribute about equally to this expansion, but it should be kept in mind that parallel diffusion is present only for numerical stability reasons. In reality, the expansion should be purely convective. The origin of the convective expansion is worth being discussed. One can see in Figure 9 that a structure of $v_{\|}$is created by the MGI, with $v_{\|} \boldsymbol{b}$ pointing away from the overdensity. This parallel flow is presumably driven by a pressure gradient resulting from the heating by parallel thermal conduction of the overdense region faster than its cooling by energy loss terms related to atomic physics. A similar phenomenon is observed in JOREK pellet injection simulations. ${ }^{39}$

It is important to set simulation parameters such that the increase in $n_{e}$ be consistent with experimental observations. In order to do this, we use synthetic interferometry. In JET, the interferometer is installed $180^{\circ}$ away toroidally from DMV2 (see Figure 10). Figure 11 shows experimental and simulated line-integrated densities for Lines of Sight (LoS) 2, 3, and 4 of the interferometer (see Figure 10 to visualize their location). Three simulations are shown, with $P_{D M V 2}=1,2$ and 5 bar, respectively. Although it does not go very far, the simulation with the experimental pressure $P_{D M V 2}=5$ bar gives a too large increase when $P_{D M V 2}=1$ or 2 bar gives a better match. This is remindful of experimental observations on the mixing efficiency of MGI (defined as the number of atoms delivered to the plasma divided by the

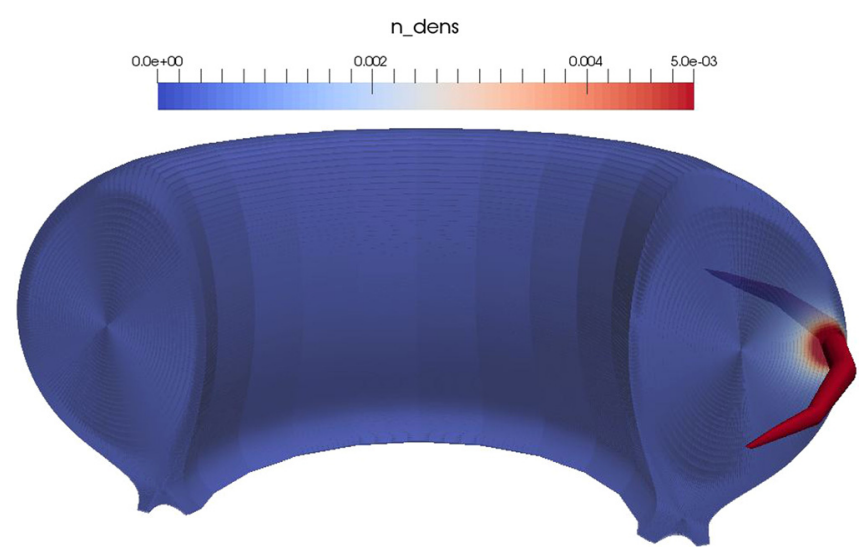

FIG. 6. Neutral density at the beginning of the MGI, in JOREK units 
Neutral density (in $10^{\wedge} 20 \mathrm{~m}-3$ )

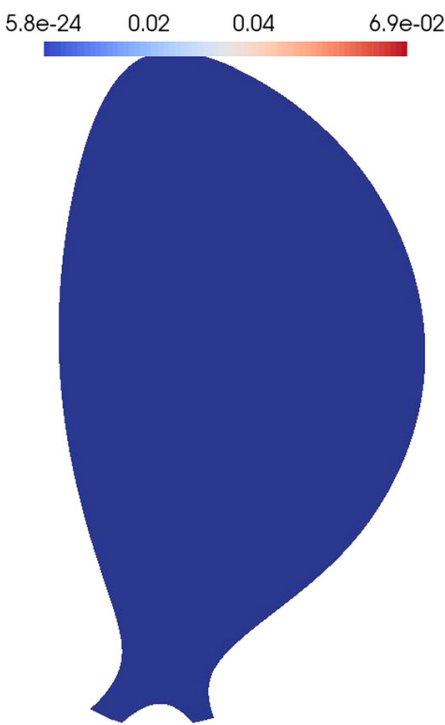

Neutral density (in $10^{\wedge} 20 \mathrm{~m}-3$ )

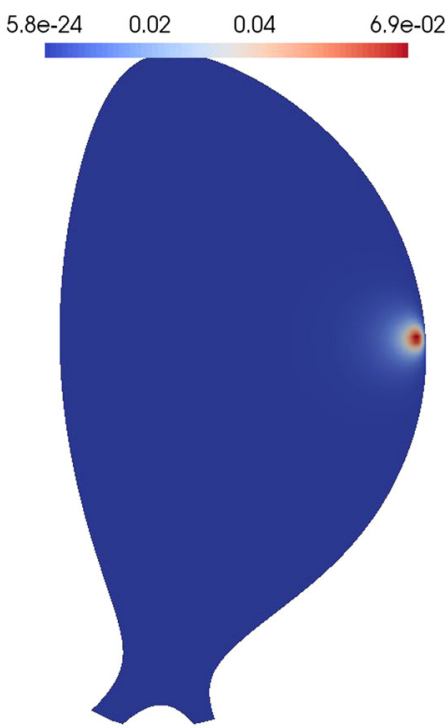

Electron density (in $10^{\wedge} 20 \mathrm{~m}-3$ )

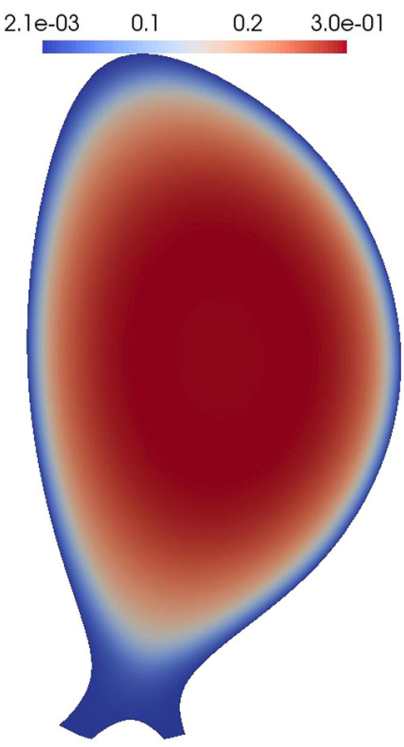

(a)

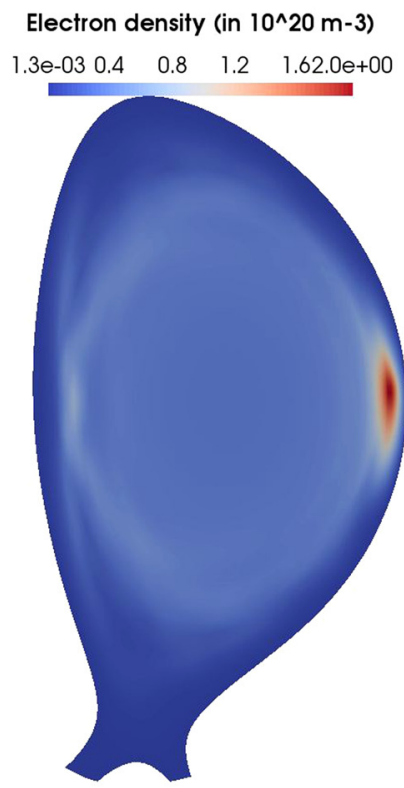

Electron temperature (in $\mathrm{keV}$ )

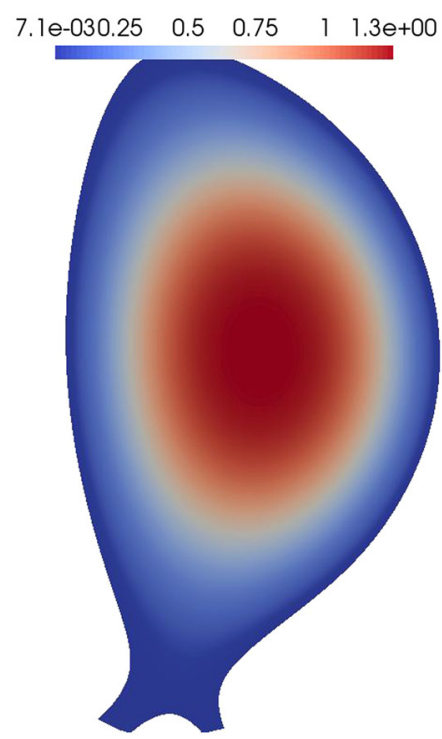

Electron temperature (in $\mathrm{keV}$ )

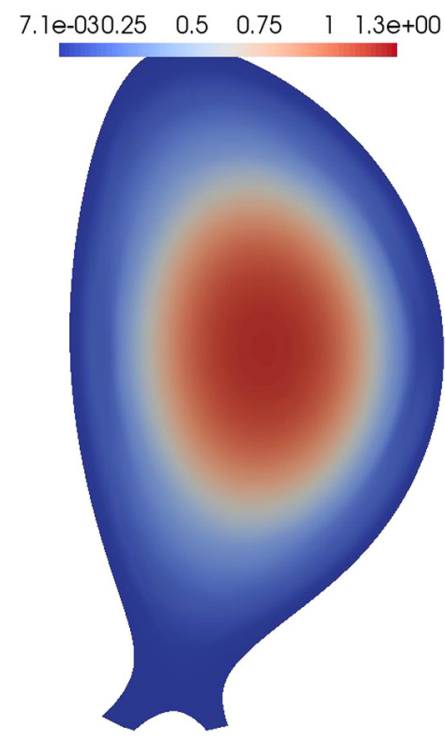

(b)

FIG. 7. Poloidal cross-sections, in the plane of the gas entry point, before (top row) and during (bottom row) the MGI, of the neutral density (left column), electron density (middle column), and electron temperature (right column).

number of atoms that have entered the vessel at a given time) which has been found of the order of a few tens of $\%$ in a range of experiments. ${ }^{13}$ The simulations described in detail in the following use $P_{D M V}=1$ bar. Looking at Figure 11, LoS 2 and 3, which are rather central, are moderately well matched with $P_{D M V 2}=1$ or 2 bar, while for $\operatorname{LoS} 4$, which goes through the edge of the plasma, the simulated value is much lower than the measured one. We found that reducing $D_{\perp}$ improves the overall match on the three LoS; however, as stated above, it tends to cause numerical instabilities.

\section{B. MHD aspects and role of $q_{0}$}

We now describe the MHD activity caused by the MGI. A particular focus is given on the role of the initial safety factor on the magnetic axis, $q_{0}$. The value given by EFIT $10 \mathrm{~ms}$ before the MGI is $q_{0}=0.78$. The fact that $q_{0}<1$ is consistent with the presence of sawteeth in this discharge. However, EFIT is not constrained by polarimetry nor motional Stark effect measurements in this pulse, thus the value of $q_{0}$ should be taken with caution. Therefore, simulations have been run with 3 values of $q_{0}: 0.75,0.94$, and 1.04 . This was done by changing the $j_{\text {mean }}$ profile while keeping $I_{p}$ 

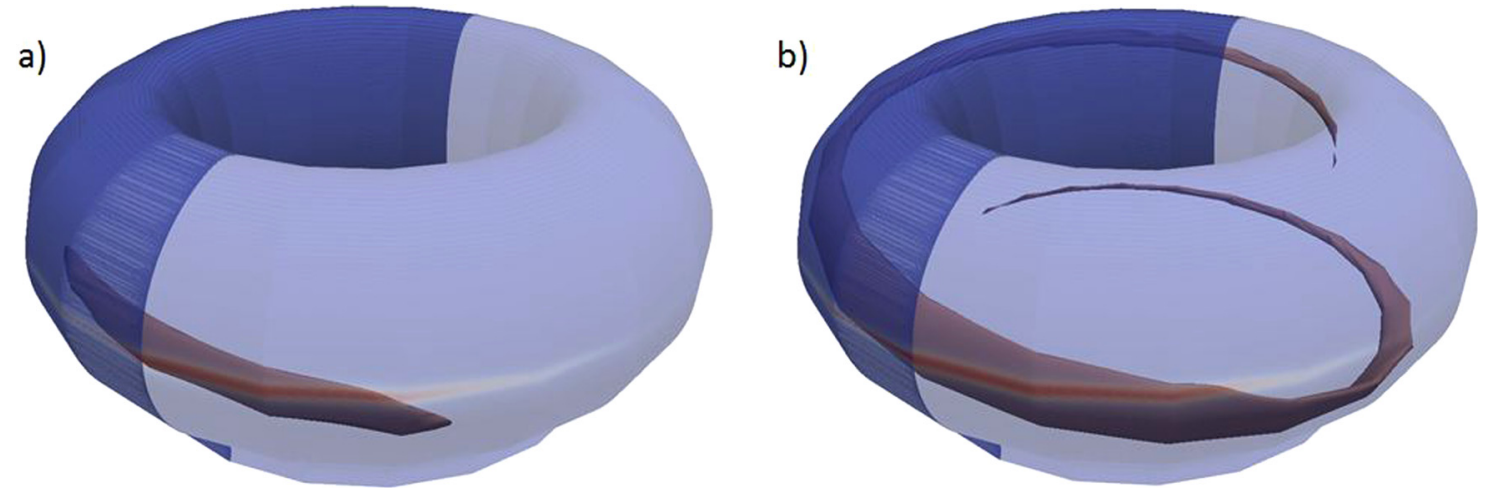

FIG. 8. Isocontours of the electron density at (a) $t=0.55 \mathrm{~ms}$ and (b) $t=0.76 \mathrm{~ms}$, showing the parallel expansion of the overdensity created by the MGI.

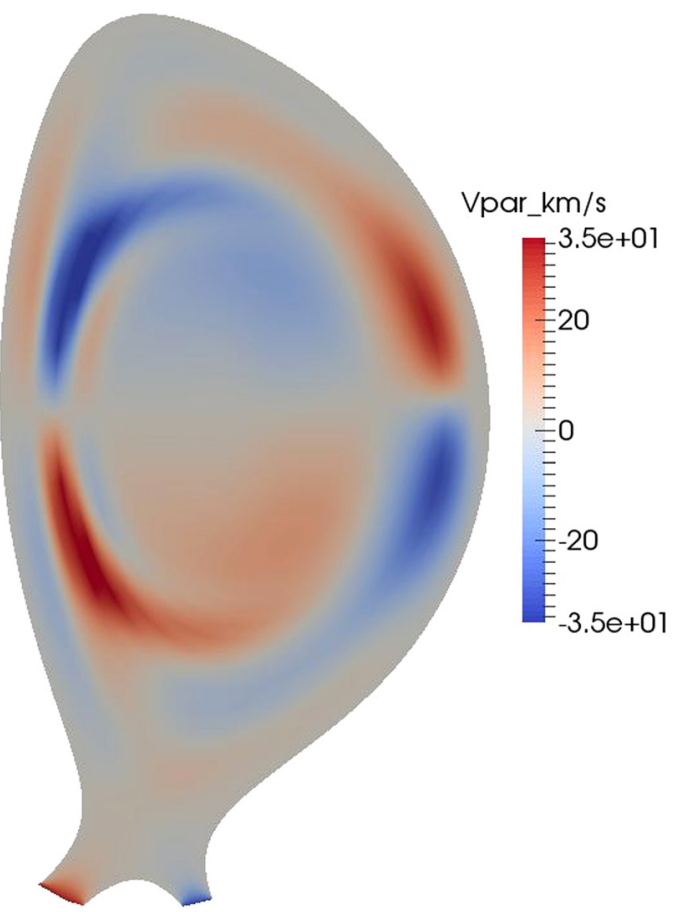

FIG. 9. Poloidal cross-section, in the plane of the gas entry point, of the parallel velocity. (almost) constant. From SXR measurements, the sawtooth inversion radius (which should give the position of the $q=1$ surface) is about $r / a=0.3$ in the sawteeth preceding the MGI. The $q_{0}=0.94$ case has the $q=1$ surface near this radius and may therefore be considered as the most realistic case. The simulations presented in this section all have $P_{D M V 2}=1$ bar. The case with $q_{0}=0.75$ has an initial central resistivity (in JOREK units) of $\eta_{0}=10^{-7}$ while the other cases have $\eta_{0}=10^{-8}$.

Figures 12-14 display time traces of the magnetic energies in the different toroidal harmonics in the three simulations (note the different time axes).

In all cases, a fast increase of the magnetic energies of all toroidal harmonics can be observed during the first millisecond or so. This increase is associated to the growth of magnetic islands, mainly $m / n=2 / 1,3 / 2$, and $1 / 1$ (the latter only for cases with $q_{0}<1$ ), all of which are visible in the Poincare cross-sections shown in Figure 15. The 1/1 mode (for simulations with $q_{0}<1$ ) is different from other modes because it is unstable even without MGI (as one would expect), as can be seen in Figure 16, which compares the magnetic energy in the $n=1$ harmonic for cases with and without MGI. The energy grows in both cases, but in the case without MGI, it starts from a very low level (numerical noise) and hence takes much longer to reach a significant

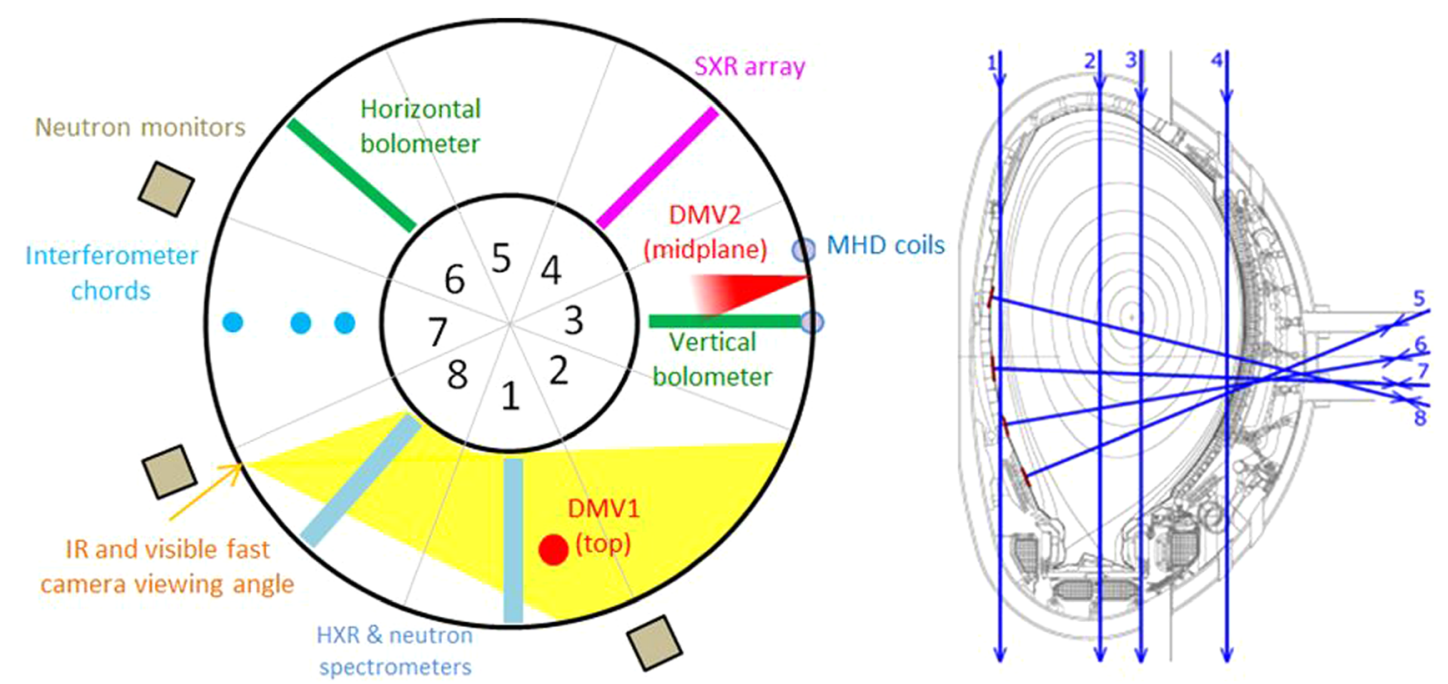

FIG. 10. Left: Location of diagnostics, DMVs and octant numbers in JET, seen from the top. Right: Interferometer lines of sight. 


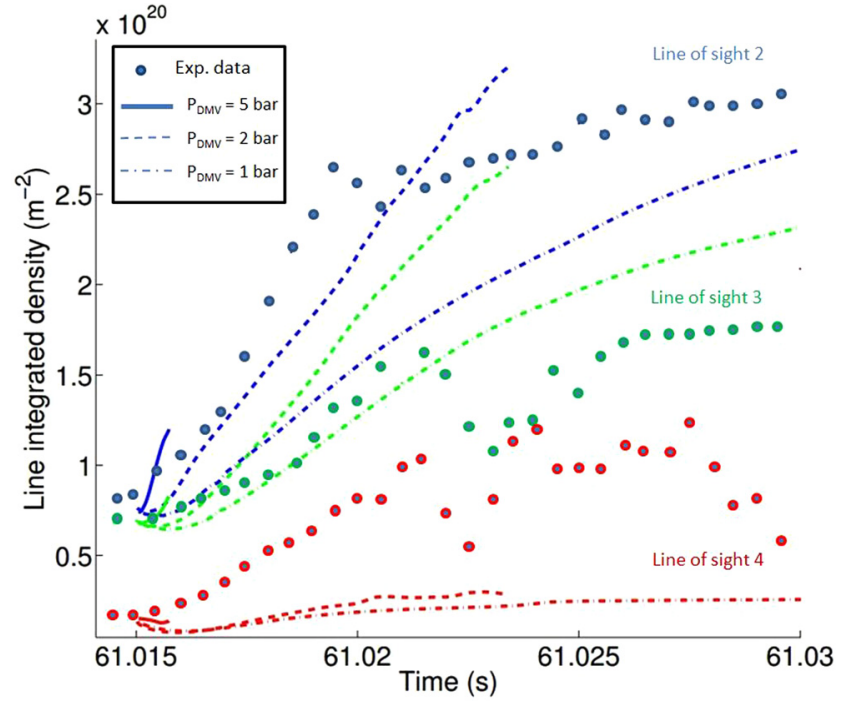

FIG. 11. Experimental and simulated interferometry measurements for 3 lines of sight, with a scan of $P_{D M V}$ in the simulations.

amplitude, while in the other case, it is seeded by the MGI and takes a much larger value from the beginning of the simulation.

It can be observed in Figure 15 that O-points of all island chains are located at the outer midplane $(\theta=0)$, i.e., in front of the MGI. This is consistent with experimental observations based on measurements with the set of saddle loops. Note that NIMROD simulations also find that the O-point of the $1 / 1$ mode is in front of the MGI location. ${ }^{21}$

Although a detailed analysis would be needed in order to understand what happens during this first phase of the simulations, the simultaneous growth of the energies of all harmonics suggests that the MGI drives the modes by directly imposing a 3D structure rather than by making the axisymmetric profiles unstable. A possible mechanism may be that the local cooling caused by the MGI reduces the toroidal current density $j$ locally through an increased

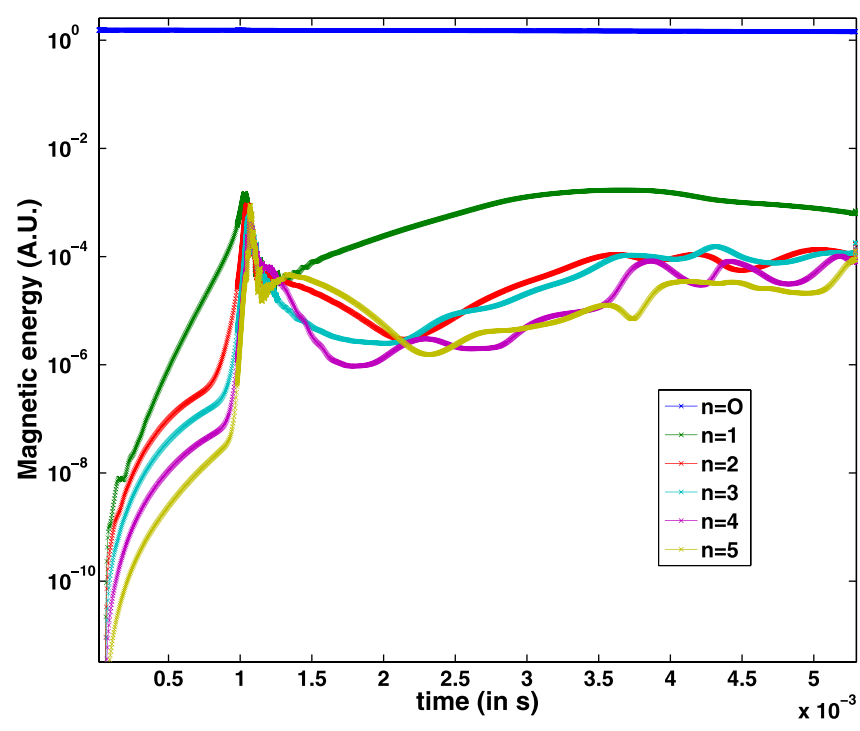

FIG. 12. Magnetic energies in the different toroidal harmonics for the simulation with $q_{0}=0.75$.

resistivity. The missing current would then cause the appearance of magnetic islands, with O-points at the position of the missing current (as in neoclassical tearing modes). The same current perturbation would also cause a magnetic perturbation $\delta \boldsymbol{B}$ in the core of the plasma which would give rise to a $\boldsymbol{j} \times \delta \boldsymbol{B}$ force pointing away from the MGI deposition region, consistently with the observed phase of the $1 / 1$ mode. This simple picture has the interest of being consistent with the observed spatial phase of the modes; however, a close look at the simulations results indicates that the reality is probably more complex. Another possibly important mechanism, for example, is that the MGI creates a 3D perturbation in the pressure field, to which $\boldsymbol{j}$ and $\boldsymbol{B}$ have to adapt in order for force balance to pertain.

In simulations with $q_{0}<1$, a crash of the $1 / 1$ mode can be observed at $t \simeq 1.2 \mathrm{~ms}\left(q_{0}=0.75\right)$ and $t \simeq 1.6 \mathrm{~ms}$ $\left(q_{0}=0.94\right)$ (see Figures 12 and 13). The crash is preceded

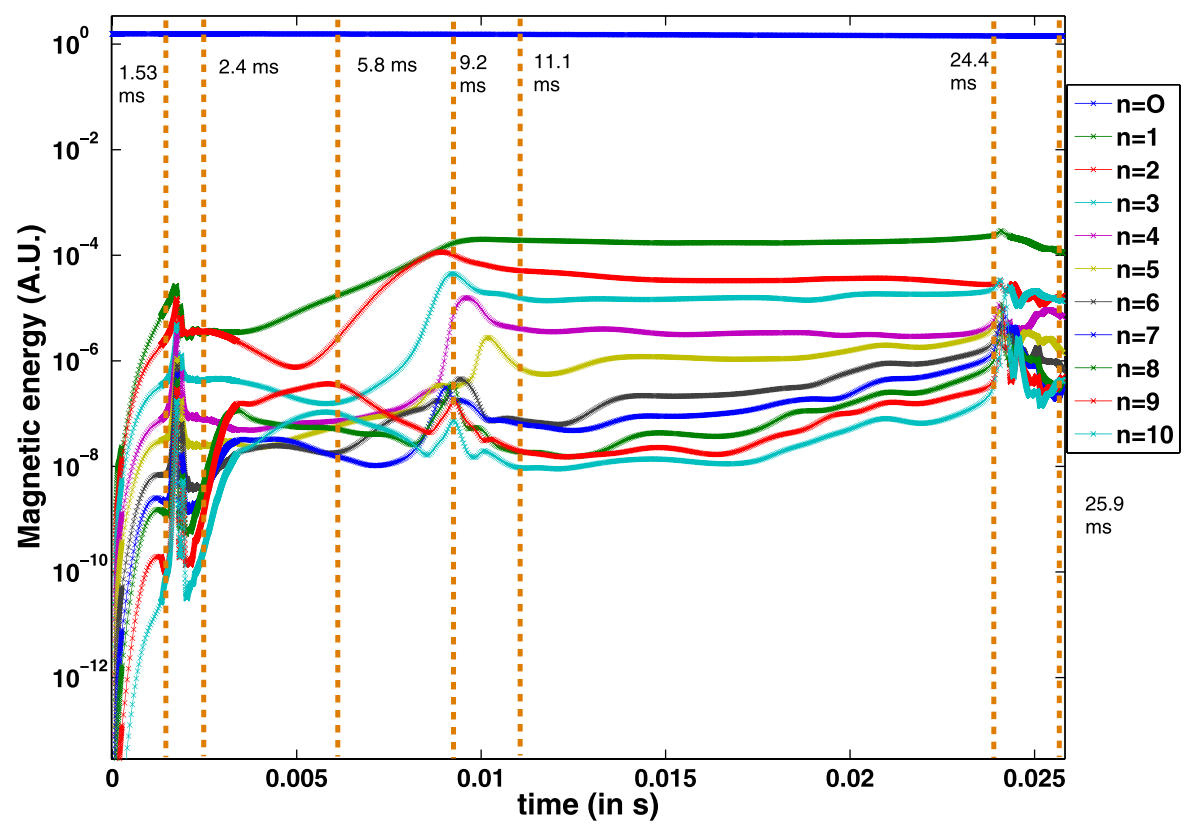

FIG. 13. Magnetic energies in the different toroidal harmonics for the simulation with $q_{0}=0.94$. 


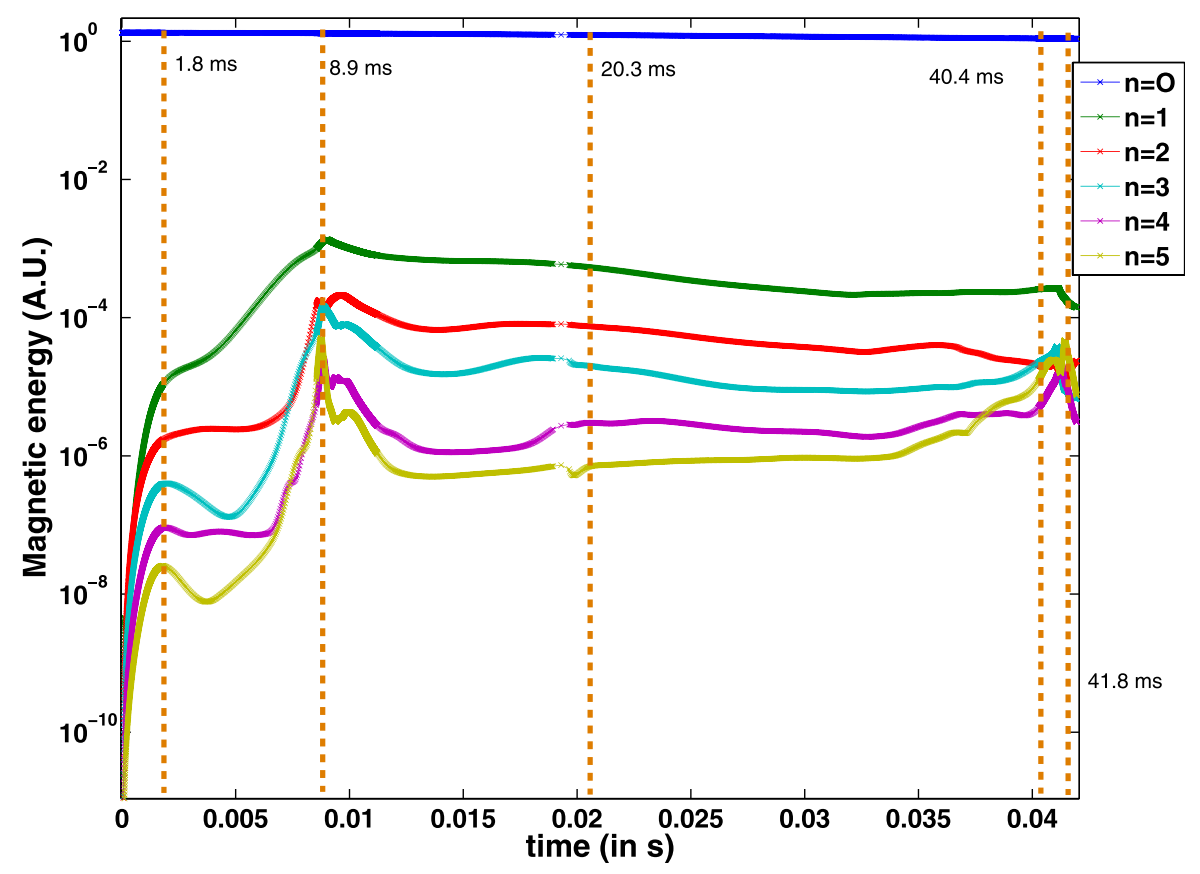

FIG. 14. Magnetic energies in the different toroidal harmonics for the simulation with $q_{0}=1.04$.

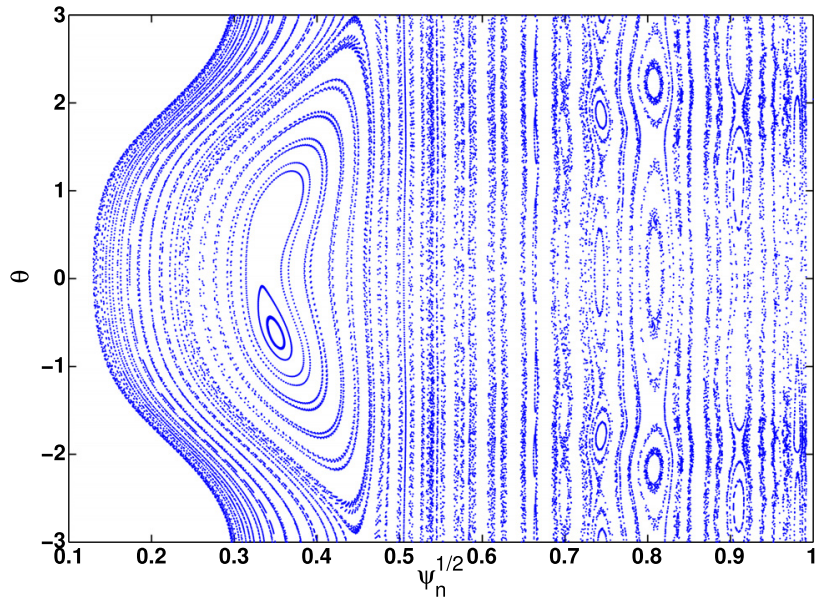

(a)

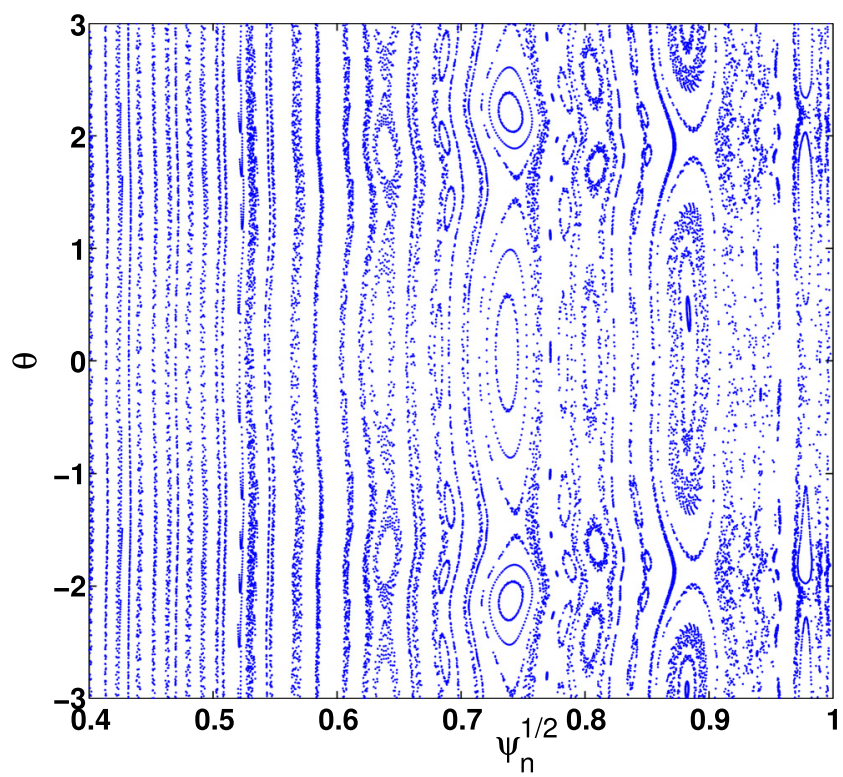

(b)

FIG. 15. Poincare cross-sections after $1.53 \mathrm{~ms}$ for the $q_{0}=0.94$ case (top) and after $1.8 \mathrm{~ms}$ for the $q_{0}=1.04$ case (bottom). by a fast growth of high $n$ harmonics, all harmonics reaching a comparable amplitude at the time of the crash, which is typical of the non-linear phase of the internal kink mode. ${ }^{40,41}$ It is interesting to compare the simulations and experiment in terms $d B / d t$ measurements from Mirnov coils. This is done in Figure 17, where it appears that the burst of $d B / d t$ in the experiment is $13 \mathrm{~ms}$ after the DMV2 trigger, which is much later than the crash of the $1 / 1$ mode in the simulations. It is not clear experimentally whether there exist signs of a $1 / 1$ mode crash near the same time as in the simulations. What is clear is that fluctuations on the same order as in the $q_{0}=0.75$ simulation are not observed at this time. As stated above, the inversion radius of sawteeth is consistent with the $q_{0}=0.94$ case, while the $q_{0}=0.75$ has the $q=1$ surface much further out. The fact that the latter case produces very large magnetic fluctuations which are not observed experimentally is therefore not surprising and merely confirms that

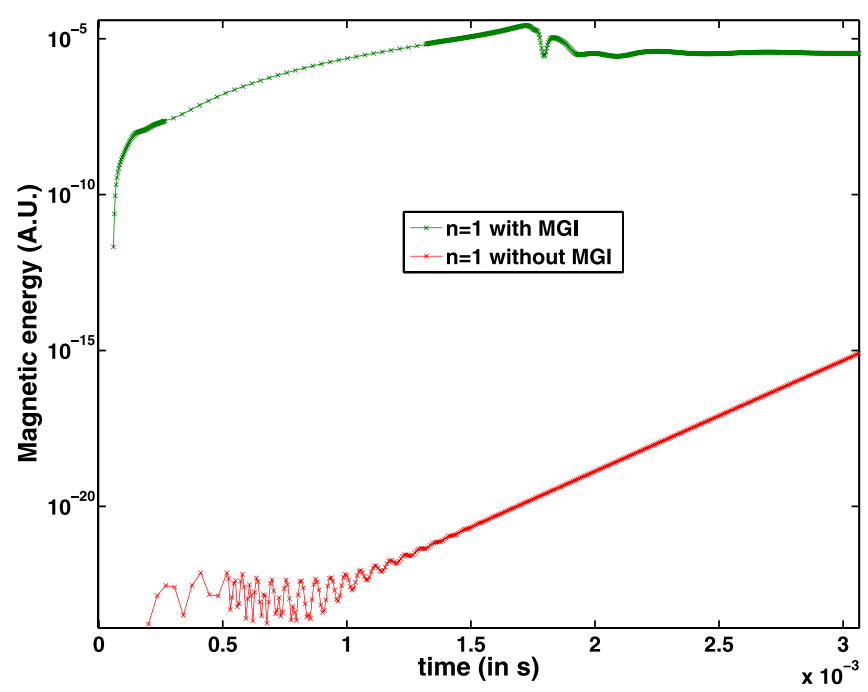

FIG. 16. Magnetic energy of the toroidal harmonic $n=1$ for the simulation with $q_{0}=0.94$, with and without MGI. 


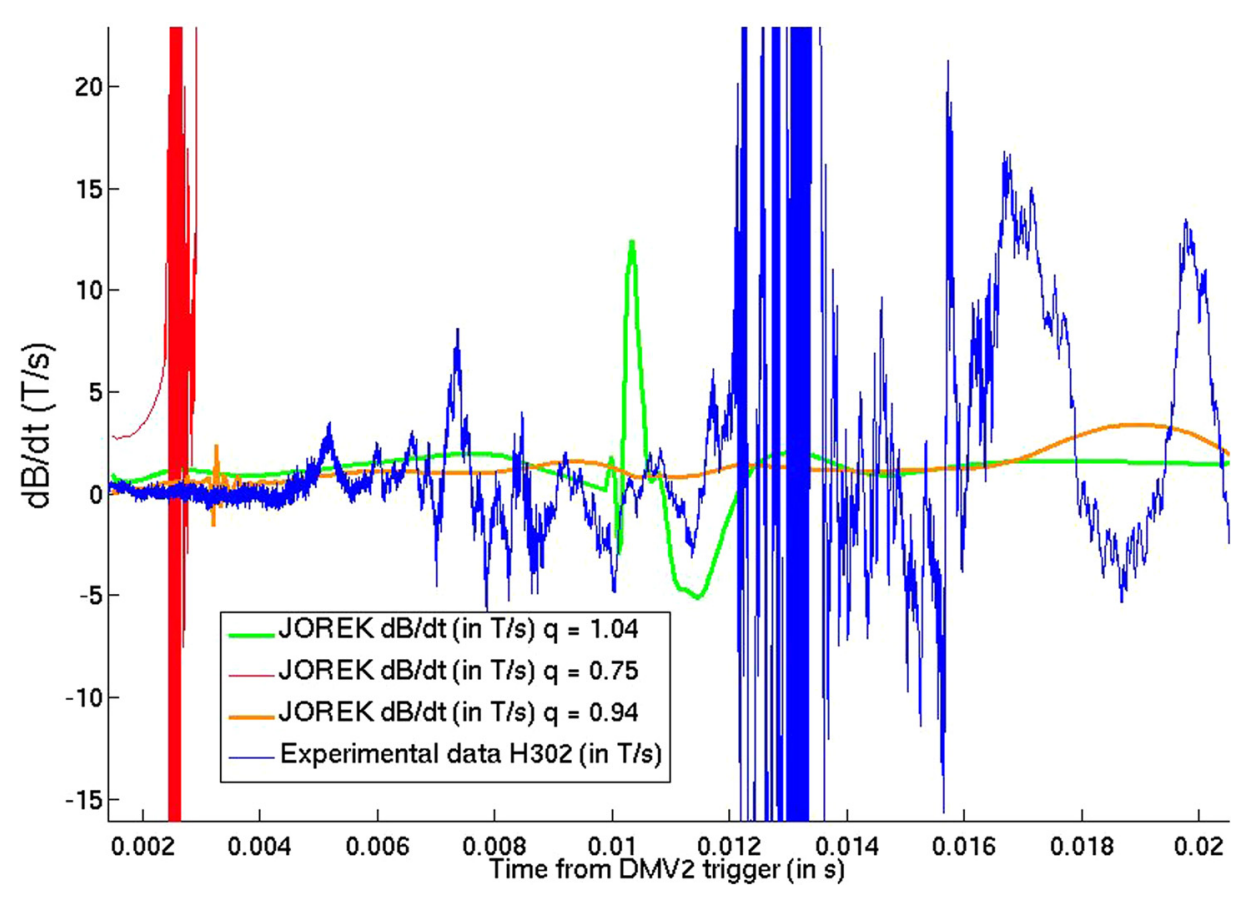

FIG. 17. Magnetic fluctuations: the red, orange, and green curves are the JOREK output for the 3 simulations and in blue is the Mirnov coil (H302) experimental datum. $t=0 \mathrm{~s}$ corresponds to the time of the DMV2 opening. The synthetic diagnostic is not fully realistic since the actual Mirnov coil (H302) is outside the JOREK computation domain. Also, the ideal wall boundary conditions may reduce the simulated $d B / d t$, since the boundary of the JOREK domain is inside the actual wall.
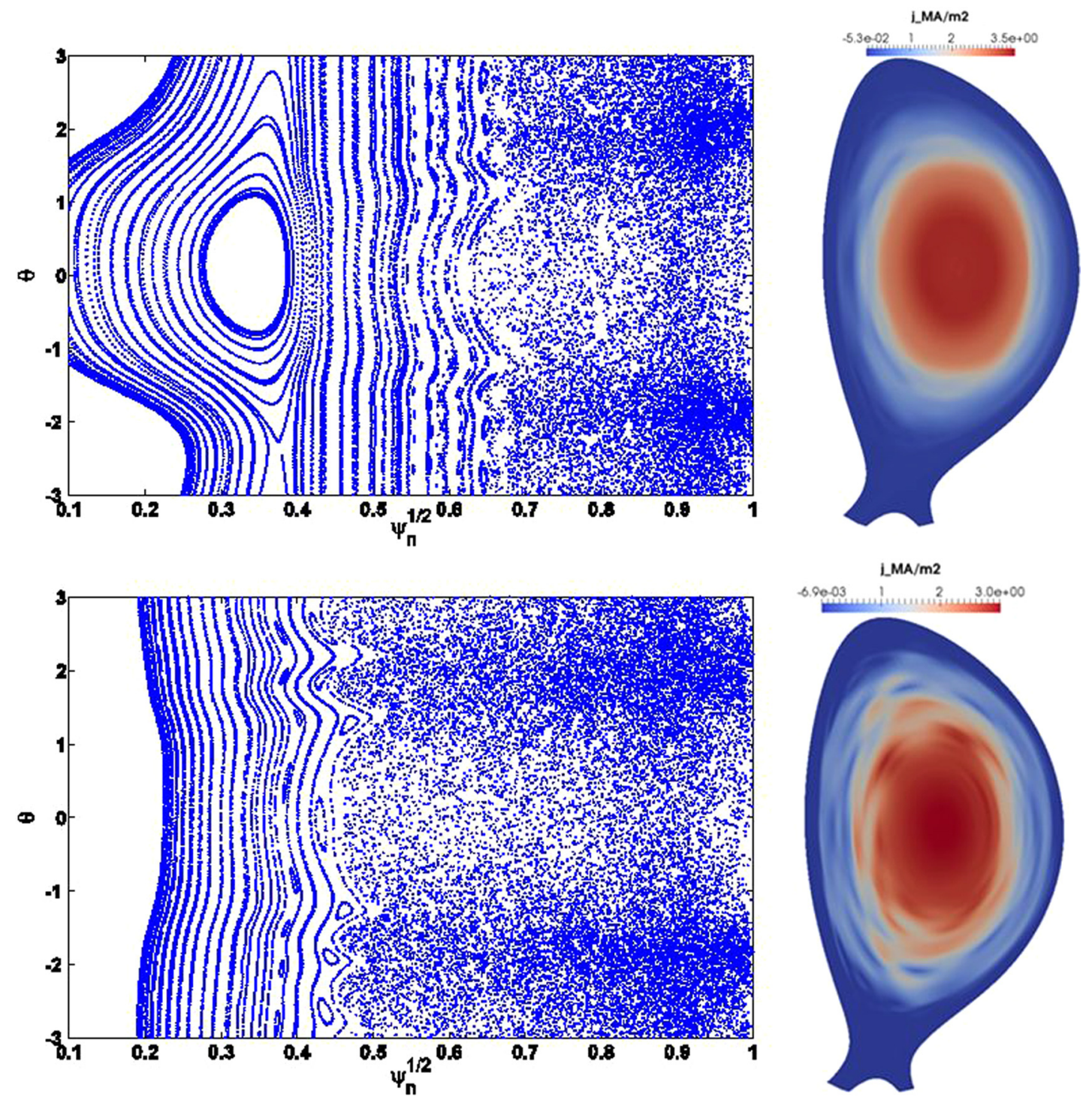

FIG. 18. Poincare cross-section and current density at the peak of MHD activity for the simulations with $q_{0}=0.94$ (upper plots) at $t=9.2$ ms and $q_{0}=1.04$ (lower plots) at $t=8.9 \mathrm{~ms}$. 
this case is not realistic. In the following, we will therefore focus on the $q_{0}=0.94$ and $q_{0}=1.04$ cases.

The second phase of the simulations, between 2 and $10 \mathrm{~ms}$ roughly, is characterised by a slower evolution of the magnetic energies. Taking a close look at Figures 13 and 14, one can see that after a short plateau-like phase between 2 and $3 \mathrm{~ms}$, the $n=1$ energy starts to increase again. Higher $n$ harmonics follow. In the $q_{0}=0.94$ case in particular, it is interesting to see that $n=2,3,4$, and 5 harmonics start to grow successively. This growth is associated to an increase in the width of magnetic islands which leads to the formation of a stochastic layer at the edge of the plasma and to small scale structures visible, for example, on $j$ (see Figure 18). A peak of MHD activity is reached around $9 \mathrm{~ms}$ (see Figures 13 and 14). The non-simultaneous growth of the energies in the different harmonics, which contrasts with the first phase of the simulations, suggests that in this second phase, the growth of the modes is due to the axisymmetric profiles becoming unstable. An often described picture ${ }^{40}$ is that MGI contracts the current channel by cooling the edge of the plasma, making it more resistive. The loss of current at the edge induces current in the still hot region inside the cold front, creating a large current gradient which can strongly drive tearing modes, especially when it is located just inside low order rational surfaces, for example, $q=2$. This effect has been found in previous JOREK simulations, leading to the TQ. ${ }^{33}$ In the present simulations, this mechanism is probably at play too. The successive growth of the $n=1,2,3,4$, and 5 harmonics in the $q_{0}=0.94$ case may be due to the successive destabilization of the $2 / 1,3 / 2,4 / 3,5 / 4$, and $6 / 5$ modes as the cold front penetrates inward. Looking at the plasma current (Figure 19), a small spike appears at $t \simeq 10$ $\mathrm{ms}$ in the $q_{0}=1.04$ simulation. At the same time, a burst of magnetic fluctuations is visible for this simulation (see Figure 17). On the other hand, neither the $I_{p}$ spike nor the burst in $d B / d t$ are distinguishable in the simulation with

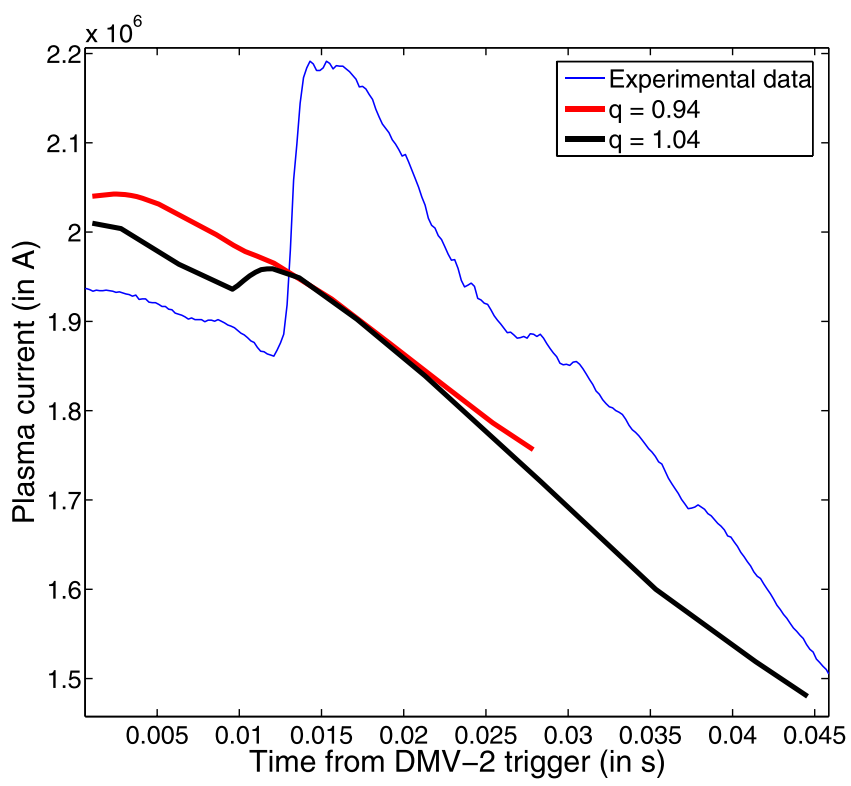

FIG. 19. Total plasma current for $q=0.94$ and $q=1.04$ cases and comparison to the experiment. $q_{0}=0.94$, which is probably related to the smaller extent of the stochastic layer and smaller magnetic energies in this case. Experimentally, both the $I_{p}$ spike and the $d B / d t$ burst are observed at about the same time as in the $q_{0}=1.04 \mathrm{sim}$ ulation, which is encouraging, but they are about one order of magnitude larger, indicating that the MHD activity in the simulations is much smaller than in the experiment. Reasons for this discrepancy will be investigated in future work. It is likely that producing a sharper current profile, possibly via a sharper cold front, would strengthen the MHD.

The third and last phases of the simulations are characterized by a much slower evolution of the energies for a few tens of millisecond, until a small burst of activity happens at $t \simeq 23 \mathrm{~ms}$ for $q_{0}=0.94$ and $t \simeq 41 \mathrm{~ms}$ for $q_{0}=1.04$. This burst is associated to the crash of a $1 / 1$ mode which can come into existence due to an increase in $j$ and drop in $q$ at the center of the plasma.

Figure 20 displays the time evolution of the central $T_{e}$ and pressure for the simulations with $q_{0}=0.94$ and $q_{0}=1.04 . T_{e}$ drops from about $1.2 \mathrm{keV}$ to about $500 \mathrm{eV}$ in
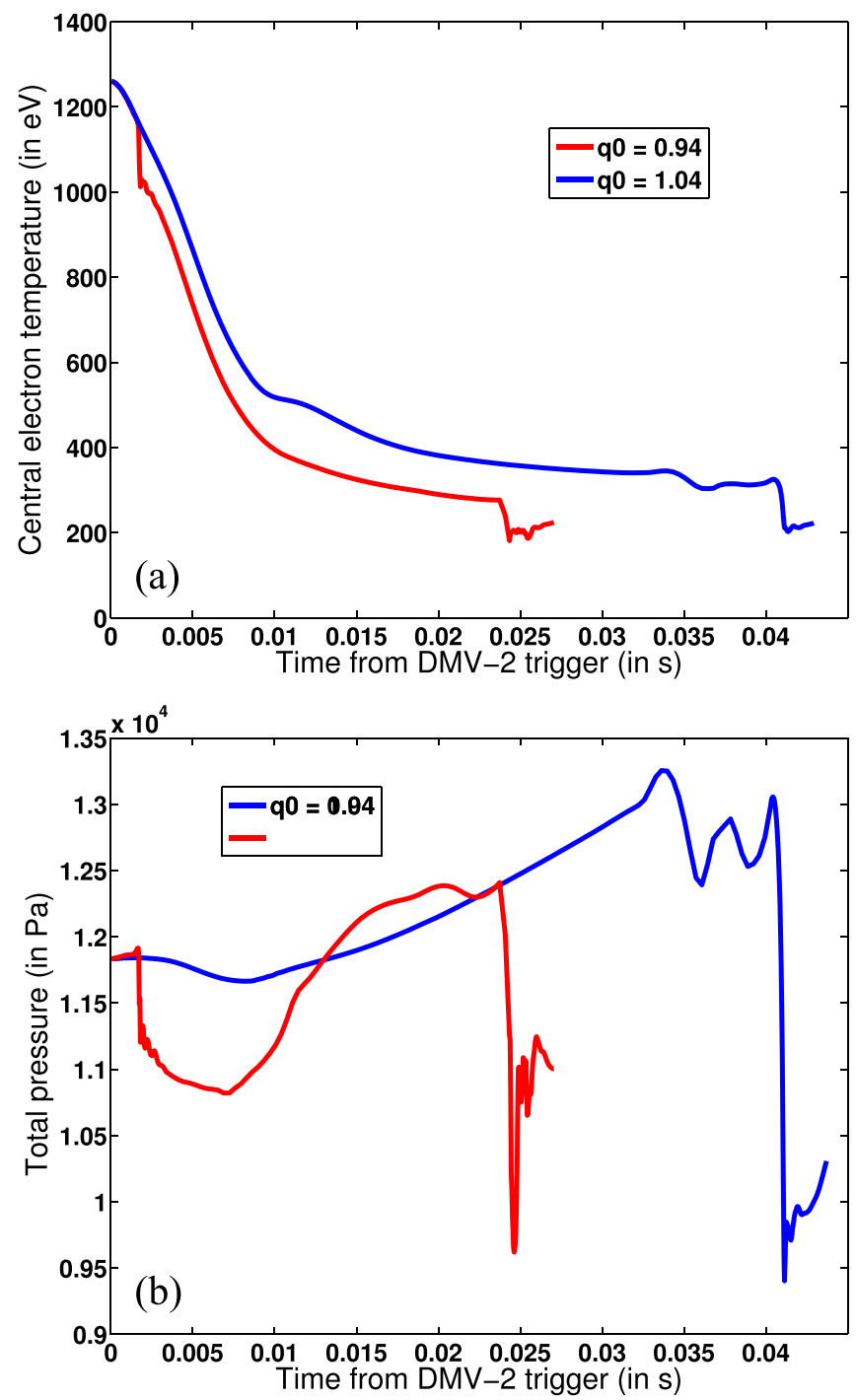

FIG. 20. Central electron temperature and central total pressure for $q=0.94$ and $q=1.04$ cases. 

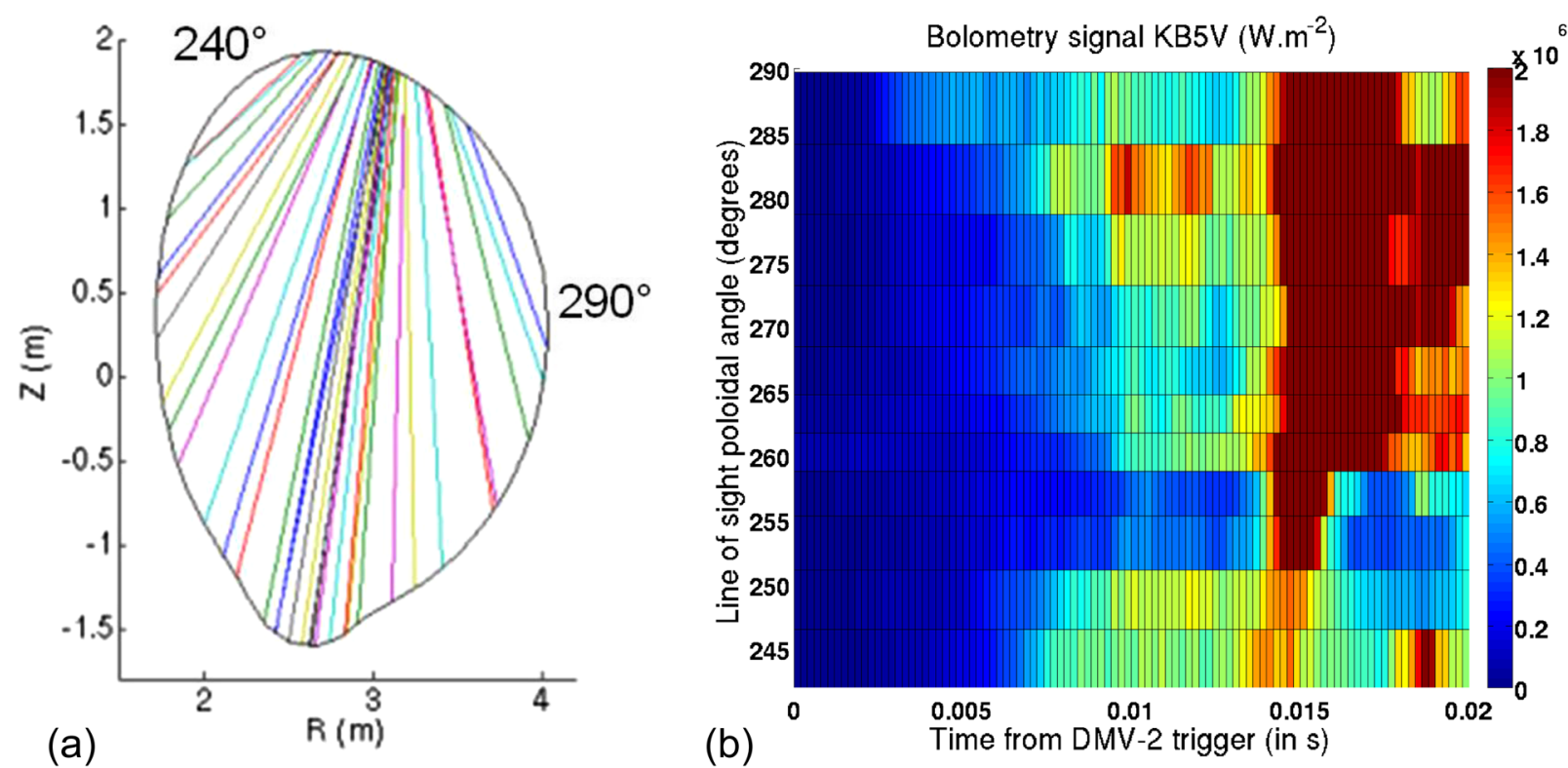

FIG. 21. Vertical bolometer measurements.

the first $10 \mathrm{~ms}$ and then decreases in a much slower way, except for the fast drops corresponding to $1 / 1$ mode crashes. It can be seen that the pressure changes much less than $T_{e}$ (see Figure 20). This is because the central cooling is mainly due to dilution, which is itself due to the perpendicular diffusion of the overdensity caused by the MGI. As mentioned in Sec. IV, a large perpendicular diffusivity is used in these simulations: $D_{\perp, S I}=28 \mathrm{~m}^{2} / \mathrm{s}$, hence the typical particle diffusion time across the plasma is on the order of $10 \mathrm{~ms}$. The fact that $T_{e}$ does not go below a few hundreds of $\mathrm{eV}$ in the simulations shows that the TQ is not fully reproduced. This is not surprising since, as we saw above, the MHD activity is much weaker in the simulations than in the experiment. In particular, the stochastic region in the simulations is confined to the outer half of the plasma, while good flux surfaces remain in the inner half. Another possible cause for the incompleteness of the TQ in the simulations is a too low level of radiation, as we shall see in the following section.

\section{Radiation}

Another key aspect of MGI-triggered disruption physics is radiation. It is also a critical aspect of the design of the ITER DMS, since a too localized radiation could result in wall melting. ${ }^{4}$ It is therefore important that simulations reproduce the measured radiation. The radiated power is measured at JET by two bolometer arrays: a vertical one located at the same toroidal angle as DMV2 and a horizontal one located $135^{\circ}$ away from it toroidally. The position of the bolometers and their LoS are shown in Figures 10, 21, and 22.
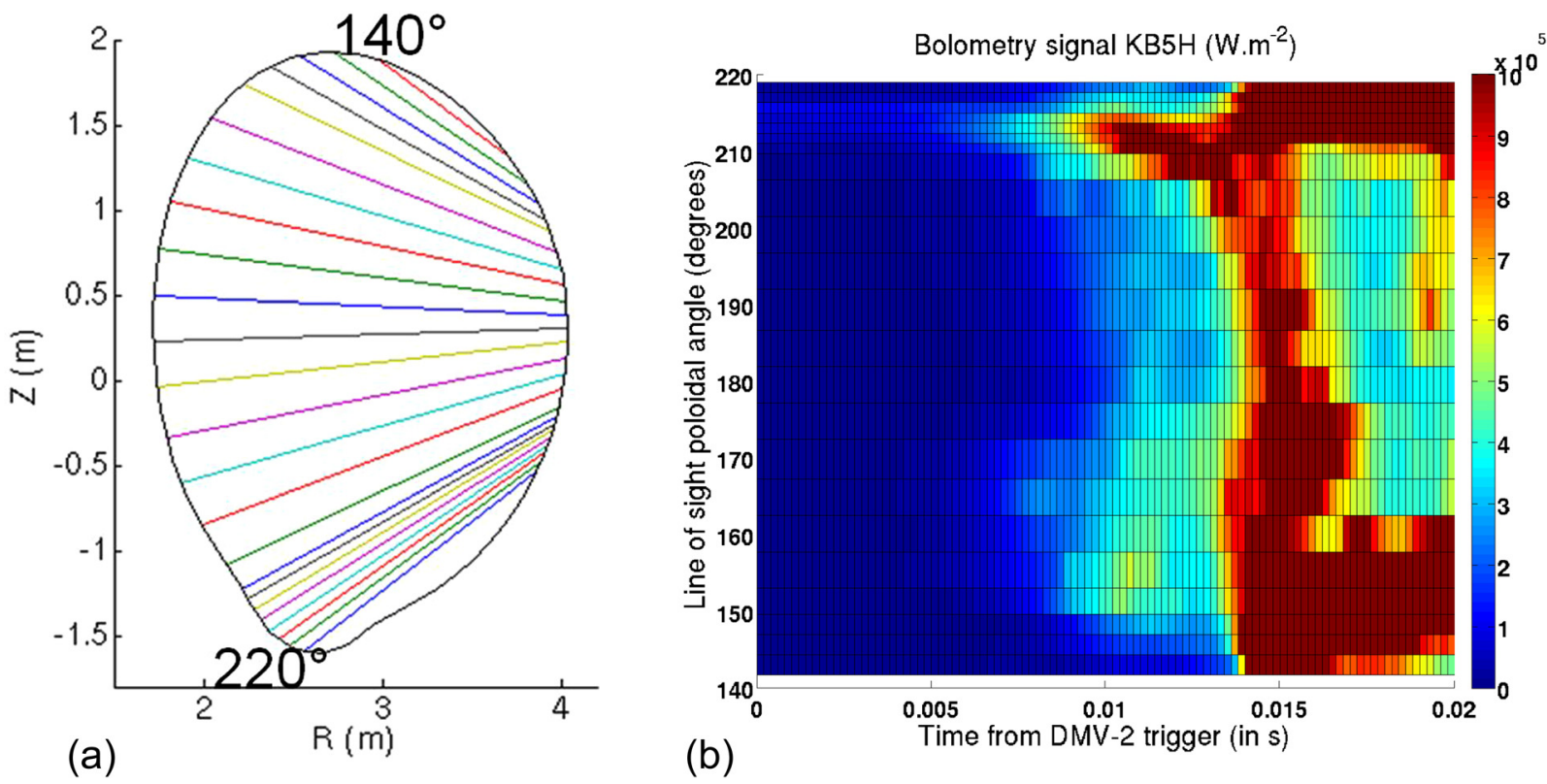

FIG. 22. Horizontal bolometer measurements. 
Figures 21 and 22 show the radiation measured by the vertical and horizontal bolometers, respectively, as a function of time and LoS poloidal angle. The first effects of the MGI are visible from $t=7 \mathrm{~ms}$ and a strong burst on virtually all LoS is visible at $t \simeq 15 \mathrm{~ms}$, corresponding to the spike in $P_{\text {rad }}$ in Figure 3. The order of magnitude of the radiation measured by the bolometers is $1 \mathrm{MW} \cdot \mathrm{m}^{-2}$, which-given that a typical chord length is $1 \mathrm{~m}$-corresponds to a volumetric radiated power of $1 \mathrm{MW} \cdot \mathrm{m}^{-3}$. It is interesting to speculate on the possible origin of this radiation. As shown in Figure 1, the bremsstrahlung (resp. $D$ line) radiation rate function is of order $10^{-38}$ $-10^{-36} \mathrm{~W} \cdot \mathrm{m}^{3}$ (resp. $10^{-32}-10^{-31} \mathrm{~W} \cdot \mathrm{m}^{3}$ ), meaning that in order to reach the observed level of radiated power, $n_{e}$ (resp. $\left(n_{e} n_{D}\right)^{0.5}$ ) should be of order $10^{21}-10^{22} \mathrm{~m}^{-3}$ (resp. $\left.1-3 \times 10^{19} \mathrm{~m}^{-3}\right)$. Given the observations presented in Sec. V A, it is unlikely that $n_{e}$ rises enough for bremsstrahlung to make a significant contribution to the observed radiation. On the other hand, $D$ line radiation cannot be excluded as a significant contributor in regions where $n_{D}>10^{18} \mathrm{~m}^{-3}$. Finally, the observed radiation may well

(a)

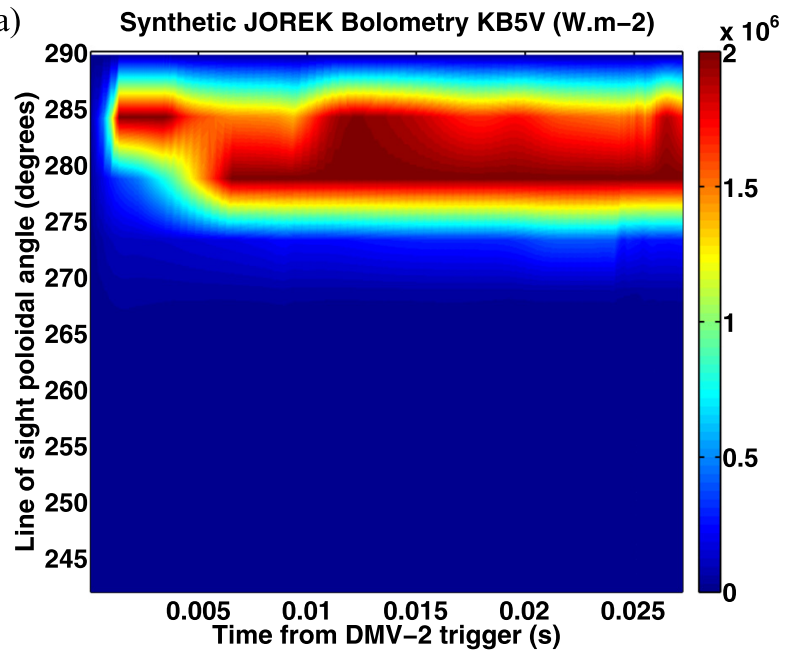

(b)

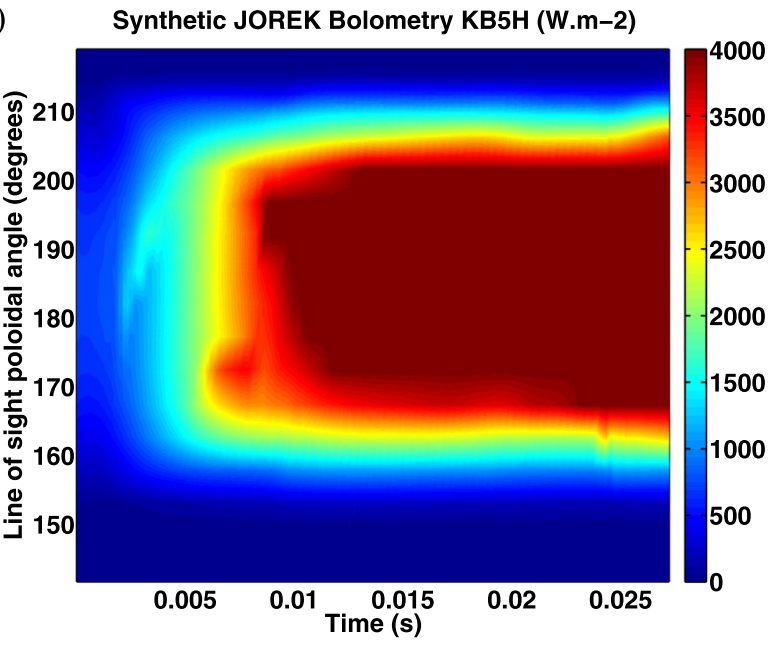

FIG. 23. Simulated bolometry signals: KB5V corresponds to the vertical bolometer and $\mathrm{KB} 5 \mathrm{H}$ to the horizontal bolometer. come from impurities, an effect which is not included in the simulations.

Synthetic bolometers have been implemented in the JOREK code, and the time evolution of the signal for each LoS is plotted in Figure 23 (for the simulation with $\left.q_{0}=0.94\right)$. Poloidal cross-sections of the bremsstrahlung and line radiated power in the toroidal plane of the bolometers are also plotted in Figure 24 to help understanding the simulation data. Experimentally, it can be noticed in Figures 21 and 22 that in the pre-TQ phase, patterns exist on the bolometry data. In particular, the horizontal bolometer (Figure 22) shows a clear peak near $212^{\circ}$ and a smoother and smaller peak near $155^{\circ}$. The $212^{\circ}$ (resp. $155^{\circ}$ ) LoS of the horizontal bolometer goes through the bottom (resp. top of the plasma) (see Figure 22). This observation may be compared to the simulated pattern of line radiation in the plane of the horizontal bolometer (bottom right plot in Figure 24), which also shows peaks in these regions (note that the line radiation peak at the outboard midplane is an artefact due to an insufficient toroidal localization of the neutral source), which are connected to the gas deposition region. The pre-TQ pattern measured by the vertical bolometer (Figure 21) is less clear but shows peaks near $262^{\circ}$ and $282^{\circ}$. The $262^{\circ}$ (resp. $282^{\circ}$ ) LoS goes through the center of the plasma and X-point region (resp. gas deposition region). In the simulations, a

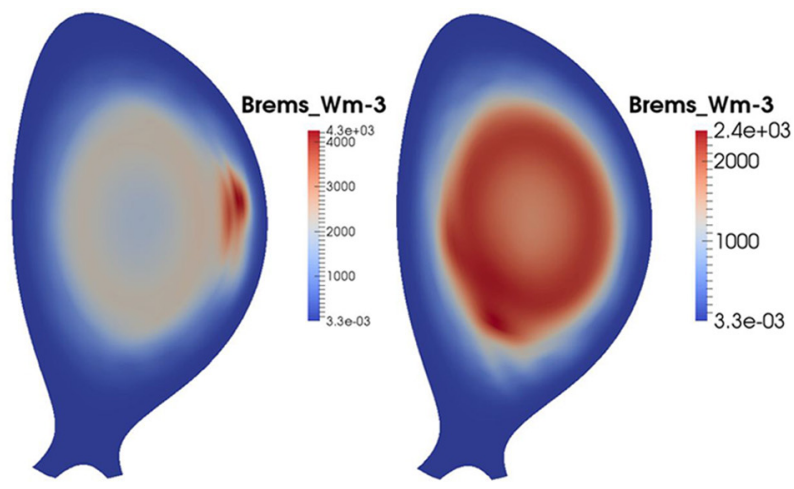

(a)

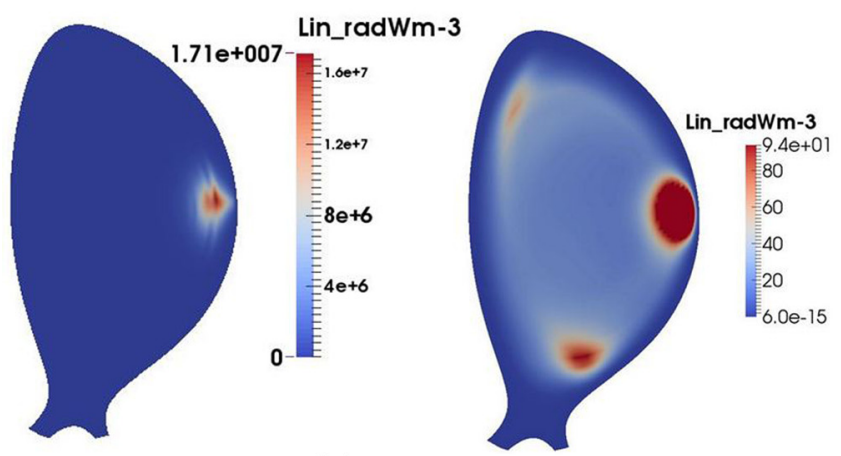

(b)

FIG. 24. Poloidal cross-sections of the bremsstrahlung (top) and line (bottom) radiated power in the toroidal plane of the vertical (left) and horizontal (right) bolometers at $t=9.35 \mathrm{~ms}$. 
strong peak also exists near $282^{\circ}$, which is dominated by line radiation in the gas deposition region where both neutral and ion densities are high (see bottom left plot in Figure 24). However, the peak near $262^{\circ}$ is not present in the simulations. It therefore appears that simulations help interpret some, but not all, qualitative features of the measured preTQ radiation pattern.

Quantitatively speaking, there is a clear mismatch for the radiation measured in the plane of the horizontal bolometer (i.e., toroidally away from DMV2). Indeed, it can be seen in Figure 24 that simulated bremsstrahlung radiation in this plane is of the order of a few $\mathrm{kW} \cdot \mathrm{m}^{-3}$ and that line radiation at the top and bottom of the plasma is even much smaller. This is by orders of magnitude smaller than measured levels (as seen comparing Figure 23 with Figures 22 and 21). We speculate that including a parallel convection term at the plasma velocity in the neutral transport equation (which may come from plasma-neutral friction by, e.g., charge exchange) would increase the line radiation in the top and bottom region and improve the match. This is planned for future work. At the location of the vertical bolometer (i.e., toroidally close to DMV2), the quantitative agreement is better in the sense that the simulated line radiation peak (see Figure 24), which is about $2 \mathrm{MW} \cdot \mathrm{m}^{-3}$, has an order of magnitude compatible with the measured peak at $282^{\circ}$ (see Figure 21).

Finally, the global radiation burst observed experimentally at $t \simeq 15 \mathrm{~ms}$ on virtually all LoS (see Figures 21 and $22)$ is absent in the simulations.

$P_{r a d}$, the total radiated power, is one order of magnitude lower than in the experiment, and the radiation burst observed in Figure 3 is not observed in the simulations.

These clear discrepancies may be due to an inappropriate gas transport model, but it seems more likely that they are due to the fact that impurities (either intrinsic or coming from the wall ${ }^{42}$ ) are not included in the present model.

\section{SUMMARY AND OUTLOOK}

First simulations of a $D_{2}$ MGI-triggered disruption in an Ohmic JET plasma have been performed with the JOREK code. The objective is to progress in the understanding of MGI-triggered disruptions, and also to validate the model on a "simple" case before applying it to more complicated cases (e.g., high Z MGI) and eventually to ITER. For this purpose, an equation for neutral density as well as appropriate atomic physics terms are included in JOREK. Present model limitations are the assumption of a purely diffusive transport of neutrals and the neglect of impurities. Other limitations come from numerical stability concerns, typically requesting diffusion coefficients to be larger than realistic.

The MGI gives rise to an overdensity that rapidly propagates in the parallel direction. Simulations with $P_{D M V 2}=1$ or 2 bar match interferometry measurements better than with the experimental value of 5 bar, suggesting that not all of the gas enters the plasma in the experiment. The main focus of the paper is on the MHD activity. In the first few milliseconds, the MGI causes the simultaneous growth of several magnetic island chains (mainly $2 / 1$ and $3 / 2$ ) and seeds the
$1 / 1$ internal kink mode in cases with $q_{0}<1$, presumably via imposing a 3D structure rather than by creating unstable axisymmetric profiles. The O-points of all islands (including $1 / 1)$ are located in front of the gas deposition region, consistently with experimental observations. In a second phase, tearing modes keep growing, but this time presumably due to an unstable current profile. A peak in MHD activity takes place near $10 \mathrm{~ms}$, associated to a stochastic layer covering roughly the outer half of the plasma and (in one of the simulations) to a peak in $I_{p}$ and a burst of $d B / d t$ on the synthetic Mirnov coil signal. These two typical signatures of the TQ are observed experimentally near the same time, which is encouraging, but with a magnitude larger by roughly one order of magnitude. Not surprisingly, the TQ is not complete in the simulations: $T_{e}$ does not go below a few hundred $\mathrm{eV}$ at the end of the simulation (most of the drop being actually due to dilution, owing to the fast diffusion of the overdensity to the center due to the large diffusion coefficient used for numerical stability reasons). This incomplete TQ can be attributed to good flux surfaces remaining in the core but may also be related to missing radiation. Indeed, the level of radiation in the simulations is much smaller than the experimental one.

In view of these results, directions for progress can clearly be identified. In order to get a complete TQ in the simulations, a much stronger MHD activity is needed. The creation of a strongly unstable current profile by the penetration of a cold front is likely to be the key. This effect has for example lead to the TQ in previous JOREK MGI simulations. ${ }^{33}$ One difference with the present simulations was that the cold front was much sharper due to different atomic physics settings. Effects that could sharpen the cold front should therefore be sought. It is interesting to assess the influence of simulation parameters. For example, we have used a $D_{\perp}$ profile with a large value only in the injection region (where it is needed for numerical stability) and a smaller value further toward the core, but this has not provided a TQ. Another possibility is that the large hyperresistivity used in this simulations for numerical reasons artificially stabilizes the MHD. This will be studied in the near future.

The observation that simulated levels of radiation are much below reality strongly suggests that a match to the experiment requests other ingredients to be added. Radiation from intrinsic impurities is a good candidate and will be added in the near future. Another direction for progress is to improve the neutral transport model, for example, by implementing neutral convection. Related to this, it may be important to take into account the momentum source associated to the MGI.

\section{ACKNOWLEDGMENTS}

We thank G. Pautasso and E. Fable for useful suggestions. This work has been carried out within the framework of the EUROfusion Consortium and has received funding from the Euratom research and training Programme 2014-2018 under Grant Agreement No. 633053. This work was granted access to the HPC resources of Aix-Marseille 
University financed by the Project Equip@Meso (ANR-10EQPX-29-01). A part of this work was carried out using the HELIOS supercomputer system (IFERC-CSC), Aomori, Japan, under the Broader Approach collaboration, implemented by Fusion for Energy and JAEA, and using the CURIE supercomputer, operated into the TGCC by CEA, France, in the framework of GENCI and PRACE projects. The views and opinions expressed herein do not necessarily reflect those of the European Commission or the ITER Organization.

${ }^{1}$ ITER Physics Expert Group on Disruptions, Plasma Control, and MHD and ITER Physics Basis Editors, Nucl. Fusion 39, 2251 (1999).

${ }^{2}$ T. C. Hender, J. C. Wesley, J. Bialek, A. Bondeson, A. H. Boozer, R. J. Buttery, A. Garofalo, T. P. Goodman, R. S. Granetz, Y. Gribov, O. Gruber, M. Gryaznevich, G. Giruzzi, S. Gnter, N. Hayashi, P. Helander, C. C. Hegna, D. F. Howel, D. A. Humphreys, G. T. A. Huysmans, A. W. Hyatt, A. Isayama, S. C. Jardin, Y. Kawano, A. Kellman, C. Kessel, H. R. Koslowski, R. J. La Haye, E. Lazzaro, Y. Q. Liu, V. Lukash, J. Manickam, S. Medvedev, V. Mertens, S. V. Mirnov, Y. Nakamura, G. Navrati, M. Okabayashi, T. Ozeki, R. Paccagnella, G. Pautasso, F. Porcelli, V. D. Pustovitov, V. Riccardo, M. Sato, O. Sauter, M. J. Schaffer, M. Shimada, P. Sonato, E. J. Strait, M. Sugihara, M. Takechi, A. D. Turnbul, E. Westerhof, D. G. Whyte, R. Yoshino, H. Zohm, and ITPA MHD, Disruption and Magnetic Control Topical Group, Nucl. Fusion 47, S128 (2007).

${ }^{3}$ A. H. Boozer, Phys. Plasmas 19, 058101 (2012).

${ }^{4}$ M. Lehnen, K. Aleynikova, P. B. Aleynikov, D. J. Campbell, P. Drewelow, N. W. Eidietis, Yu. Gasparyan, R. S. Granetz, Y. Gribov, N. Hartmann, E. M. Hollmann, V. A. Izzo, S. Jachmich, S.-H. Kim, M. Koan, H. R. Koslowski, D. Kovalenko, U. Kruezi, A. Loarte, S. Maruyama, G. F. Matthews, P. B. Parks, G. Pautasso, R. A. Pitts, C. Reux, V. Riccardo, R. Roccella, J. A. Snipes, A. J. Thornton, P. C. de Vries, and EFDA JET Contributors, "Disruptions in ITER and strategies for their control and mitigation," J. Nucl. Mater. 463, 39-48 (2015). Available at http:// www.sciencedirect.com/science/article/pii/S0022311514007594.

${ }^{5}$ E. M. Hollmann, P. B. Aleynikov, T. Flp, D. A. Humphreys, V. A. Izzo, M. Lehnen, V. E. Lukash, G. Papp, G. Pautasso, F. Saint-Laurent, and J. A. Snipes, Phys. Plasmas 22, 021802 (2015).

${ }^{6}$ M. Lehnen, A. Alonso, G. Arnoux, N. Baumgarten, S. A. Bozhenkov, S. Brezinsek, M. Brix, T. Eich, S. N. Gerasimov, A. Huber, S. Jachmich, U. Kruezi, P. D. Morgan, V. V. Plyusnin, C. Reux, V. Riccardo, G. Sergienko, M. F. Stamp, and JET EFDA Contributors, Nucl. Fusion 51, 123010 (2011).

${ }^{7}$ M. Lehnen, G. Arnoux, S. Brezinsek, J. Flanagan, S. N. Gerasimov, N. Hartmann, T. C. Hender, A. Huber, S. Jachmich, V. Kiptily, U. Kruezi, G. F. Matthews, J. Morris, V. V. Plyusnin, C. Reux, V. Riccardo, B. Sieglin, P. C. de Vries, and JET EFDA Contributors, Nucl. Fusion 53, 093007 (2013).

${ }^{8}$ C. Reux, V. Plyusnin, B. Alper, D. Alves, B. Bazylev, E. Belonohy, S. Brezinsek, J. Decker, S. Devaux, P. de Vries, A. Fil, S. Gerasimov, I. Lupelli, S. Jachmich, E. M. Khilkevitch, V. Kiptily, R. Koslowski, U. Kruezi, M. Lehnen, A. Manzanares, J. Mlynr, E. Nardon, E. Nilsson, V. Riccardo, F. Saint-Laurent, A. E. Shevelev, C. Sozzi, and JET EFDA Contributors, "Runaway beam studies during disruptions at JET-ILW," J. Nucl. Mater. 463, 143-149 (2015). Available at http://www.sciencedirect. com/science/article/pii/S0022311514006850.

${ }^{9}$ P. C. de Vries, G. Arnoux, A. Huber, J. Flanagan, M. Lehnen, V. Riccardo, C. Reux, S. Jachmich, C. Lowry, G. Calabro, D. Frigione, M. Tsalas, N. Hartmann, S. Brezinsek, M. Clever, D. Douai, M. Groth, T. C. Hender, E. Hodille, E. Joffrin, U. Kruezi, G. F. Matthews, J. Morris, R. Neu, V. Philipps, G. Sergienko, M. Sertoli, and JET EFDA Contributors, Plasma Phys. Controlled Fusion 54, 124032 (2012).

${ }^{10}$ G. Pautasso, Y. Zhang, B. Reiter, L. Giannone, O. Gruber, A. Herrmann, O. Kardaun, K. K. Khayrutdinov, V. E. Lukash, M. Maraschek, A. Mlynek, Y. Nakamura, W. Schneider, G. Sias, M. Sugihara, and ASDEX Upgrade Team, Nucl. Fusion 51, 103009 (2011).

${ }^{11}$ C. Reux, J. Bucalossi, F. Saint-Laurent, C. Gil, P. Moreau, and P. Maget, Nucl. Fusion 50, 095006 (2010).

${ }^{12}$ E. M. Hollmann, G. Arnoux, N. Commaux, N. W. Eidietis, T. E. Evans, R. S. Granetz, A. Huber, D. A. Humphreys, V. A. Izzo, A. N. James, T. C. Jernigan, M. Lehnen, G. Maddaluno, R. Paccagnella, P. B. Parks, V. Philipps, M. L. Reinke, D. L. Rudakov, F. Saint-Laurent, V. Sizyuk, E. J.
Strait, J. C. Wesley, C. P. C. Wong, and J. H. Yu, J. Nucl. Mater. 415, S27 (2011).

${ }^{13}$ S. A. Bozhenkov, M. Lehnen, K. H. Finken, G. Bertschinger, H. R. Koslowski, D. Reiter, R. C. Wolf, and TEXTOR Team, Nucl. Fusion 51 083033 (2011).

${ }^{14}$ M. Bakhtiari, G. Olynyk, R. Granetz, D. G. Whyte, M. L. Reinke, K. Zhurovich, and V. Izzo, Nucl. Fusion 51, 063007 (2011).

${ }^{15}$ C. Reux, V. Plyusnin, B. Alper, D. Alves, B. Bazylev, E. Belonohy, A. Boboc, S. Brezinsek, J. Decker, P. Drewelow, S. Devaux, P. de Vries, A. Fil, S. Gerasimov, L. Giacomelli, S. Jachmich, E. M. Khilkevitch, V. Kiptily, R. Koslowski, U. Kruezi, M. Lehnen, I. Lupelli, A. Manzanares, A. M. De Aguilera, G. Matthews, J. Mlynar, E. Nardon, E. Nilsson, V. Riccardo, F. Saint-Laurent, A. E. Shevelev, C. Sozzi, and JET contributors, "Runaway electron beam generation and mitigation during disruptions at JET-ILW," Nucl. Fusion (to be published).

${ }^{16}$ M. Sugihara, S. Putvinski, D. Campbell, S. Carpentier-Chouchana, F. Escourbiac, S. Gerasimov, Y. Gribov, T. Hender, T. Hirai, K. Ioki, R. Khayrutdinov, H. Labidi, V. Lukash, S. Maruyama, M. Merola, R. Mitteau, S. Miyamoto, J. Morris, G. Pautasso, R. Pitts, R. Raffray, V. Riccardo, R. Roccella, G. Sannazzaro, T. Schioler, J. Snipes, and R. Yoshino, in Proceedings of 24th IAEA FEC, ITR/P1-14, San Diego, USA, 2012.

${ }^{17}$ S. Putvinski, L. Baylor, D. Campbell, V. Chuyanov, Yu. Gribov, V. Leonov, A. Loarte, S. Maruyama, R. Pearce, R. A. Pitts, A. Polevoi, R. Mitteau, and M. Sugihara, in Proc. 23rd International Conference on Fusion Energy, Daejeon, Korea, 2010.

${ }^{18}$ L. Zeng, H. R. Koslowski, Y. Liang, A. Lvovskiy, M. Lehnen, D. Nicolai, J. Pearson, M. Rack, H. Jaegers, K. H. Finken, K. Wongrach, Y. Xu, and TEXTOR Team, Phys. Rev. Lett. 110, 235003 (2013).

${ }^{19}$ A. Bondeson, R. D. Parker, M. Hugon, and P. Smeulders, Nucl. Fusion 31, 1695 (1991).

${ }^{20}$ R. Paccagnella, H. R. Strauss, and J. Breslau, Nucl. Fusion 49, 035003 (2009).

${ }^{21}$ V. A. Izzo, Phys. Plasmas 20, 056107 (2013).

${ }^{22}$ V. A. Izzo, E. M. Hollmann, A. N. James, J. H. Yu, D. A. Humphreys, L. L. Lao, P. B. Parks, P. E. Sieck, J. C. Wesley, R. S. Granetz, G. M. Olynyk, and D. G. Whyte, Nucl. Fusion 51, 063032 (2011).

${ }^{23}$ V. A. Izzo, P. B. Parks, and L. L. Lao, Plasma Phys. Controlled Fusion 51, 105004 (2009)

${ }^{24}$ V. A. Izzo, D. G. Whyte, R. S. Granetz, P. B. Parks, E. M. Hollmann, L. L. Lao, and J. C. Wesley, Phys. Plasmas 15, 056109 (2008).

${ }^{25}$ V. A. Izzo, Nucl. Fusion 46, 541 (2006).

${ }^{26}$ G. T. A. Huysmans and O. Czarny, Nucl. Fusion 47, 659 (2007).

${ }^{27}$ G. T. A. Huysmans, S. Pamela, E. van der Plas, and P. Ramet, Plasma Phys. Controlled Fusion 51, 124012 (2009).

${ }^{28}$ F. Orain, M. Becoulet, G. Dif-Pradalier, G. Huijsmans, S. Pamela, E. Nardon, C. Passeron, G. Latu, V. Grandgirard, A. Fil, A. Ratnani, I. Chapman, A. Kirk, A. Thornton, M. Hoelzl, and P. Cahyna, Phys. Plasmas 20, 102510 (2013).

${ }^{29}$ M. Becoulet, F. Orain, G. T. A. Huijsmans, S. Pamela, P. Cahyna, M. Hoelzl, X. Garbet, E. Franck, E. Sonnendrcker, G. Dif-Pradalier, C. Passeron, G. Latu, J. Morales, E. Nardon, A. Fil, B. Nkonga, A. Ratnani, and V. Grandgirard, Phys. Rev. Lett. 113, 115001 (2014).

${ }^{30}$ F. Orain, M. Becoulet, G. T. A. Huijsmans, G. Dif-Pradalier, M. Hoelzl, J. Morales, X. Garbet, E. Nardon, S. Pamela, C. Passeron, G. Latu, A. Fil, and P. Cahyna, Phys. Rev. Lett. 114, 035001 (2015).

${ }^{31}$ C. Reux, "Ecole doctorale de 1Ecole Polytechnique," Ph.D. thesis (Paris Institute of Technology, 2010).

${ }^{32}$ C. Reux, G. Huysmans, J. Bucalossi, and M. Bcoulet, in Proceedings of the 38th European Physical Society Conference on Plasma Physics (European Physical Society, 2011).

${ }^{33}$ A. Fil, E. Nardon, P. Beyer, M. Becoulet, G. Dif-Pradalier, V. Grandgirard, R. Guirlet, M. Hoelzl, G. T. A. Huijsmans, G. Latu, M. Lehnen, A. Loarte, F. Orain, S. Pamela, C. Passeron, C. Reux, F. SaintLaurent, P. Tamain, and JET EFDA Contributors, in Proceedings of the 41st European Physical Society Conference on Plasma Physics, Berlin, Germany, 2014.

${ }^{34} \mathrm{G}$. S. Voronov, "A practical fit formula for ionization rate coefficients of atoms and ions by electron impact: $Z=1-28$," At. Data Nucl. Data Tables 65(1), 1-35 (1997).

${ }^{35}$ R. H. Huddlestone and S. L. Leonard, Plasma Diagnostic TechniquesSpectral Intensities (Academic Press, New York, 1965).

${ }^{36}$ See http://adas.ac.uk for: H. P. Summers, Atomic Data and Analysis Structure User Manual. 
${ }^{37}$ J. Wesson, Tokamaks (Clarendon, Oxford, 2004).

${ }^{38} \mathrm{O}$. Czarny and G. Huysmans, J. Comput. Phys. 227, 7423-7445 (2008)

${ }^{39}$ S. Futatani, G. Huijsmans, A. Loarte, L. R. Baylor, N. Commaux, T. C. Jernigan, M. E. Fenstermacher, C. Lasnier, T. H. Osborne, and B. Pegourie, Nucl. Fusion 54, 073008 (2014).
${ }^{40}$ D. Biskamp, Nonlinear Magnetohydrodynamics (Cambridge University Press, Cambridge, U.K., 2003).

${ }^{41}$ See https://tel.archives-ouvertes.fr/pastel-00926428/document for: $\mathrm{T}$. Nicolas, Sawtooth driven particle transport in tokamak plasmas, Ecole Polytechnique X, 2013.

${ }^{42}$ D. J. Ward and J. A. Wesson, Nucl. Fusion 32, 1117 (1992). 